Check for updates

Cite this: Phys. Chem. Chem. Phys. $2021,23,15440$

Received 17th March 2021, Accepted 17th June 2021

DOI: $10.1039 / \mathrm{d} 1 \mathrm{cp} 01203 \mathrm{~g}$

rsc.li/pccp

\title{
Bicolour fluorescent molecular sensors for cations: design and experimental validation
}

\author{
Zoraida Freixa, (D) *ab Iván Rivilla, (D) ac Francesc Monrabal, (D) ac \\ Juan J. Gómez-Cadenas (D) ac and Fernando P. Cossío (D)*cd
}

\begin{abstract}
Molecular entities whose fluorescence spectra are different when they bind metal cations are termed bicolour fluorescent molecular sensors. The basic design criteria of this kind of compound are presented and the different fluorescent responses are discussed in terms of their chemical behaviour and electronic features. These latter elements include intramolecular charge transfer (ICT), formation of intramolecular and intermolecular excimer/exciplex complexes and Förster resonance energy transfer (FRET). Changes in the electronic properties of the fluorophore based on the decoupling between its constitutive units upon metal binding are also discussed. The possibility of generating fluorescent bicolour indicators that can capture metal cations in the gas phase and at solid-gas interfaces is also discussed.
\end{abstract}

\section{Introduction}

${ }^{a}$ Ikerbasque, Basque Foundation for Science, 48009 Bilbao, Spain. E-mail: zoraida_freixa@ehu.eus

${ }^{b}$ Department of Applied Chemistry, Faculty of Chemistry,

University of the Basque Country (UPV/EHU), 20018 San Sebastián/Donostia, Spain

${ }^{c}$ Donostia International Physics Center (DIPC), 20018 San Sebastián/Donostia, Spain

${ }^{d}$ Department of Organic Chemistry I, Faculty of Chemistry, University of the Basque Country (UPV/EHU), 20018 San Sebastián/Donostia, Spain

Detection of metal cations by means of molecular sensors has become a very active research field, with important applications in analytical chemistry, ${ }^{1}$ materials $^{2}$ and environmental sciences, ${ }^{3}$ and biological chemistry, ${ }^{4}$ among other scientific areas. Very recently, this technique has emerged as a promising tool in particle physics since luminescence

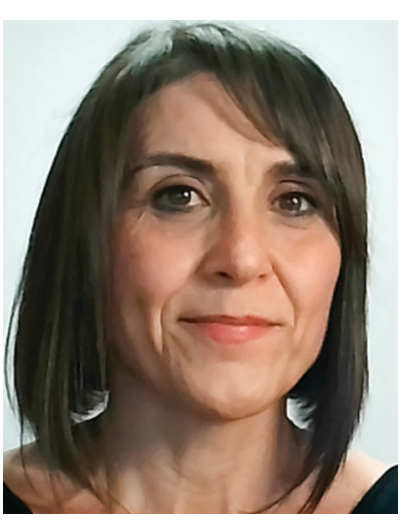

Zoraida Freixa
Zoraida Freixa completed her $P h D$ in the area of homogeneous catalysis at the Autonomous University of Barcelona. After a post-doctoral stage at Prof. van Leeuwen's group in Amsterdam, she enrolled as Ramón y Cajal Researcher at the Institute of Chemical Research of Catalonia (ICIQ) as a group manager of Prof. van Leeuwen's group. Since 2010, and after a short period as a Lecturer at the University of Barcelona, she has been holding an Ikerbasque Research Professor position at the University of the Basque Country (UPV/EHU) in San Sebastián. She leads a research group focused on the development of photoswitchable and/or chiral organometallic compounds with special emphasis on the study of their catalytic and luminescence properties. Since 2019, she has been involved in the development of bicolour sensors for barium and radium tagging in $\beta \beta 0 \nu$ reactions.

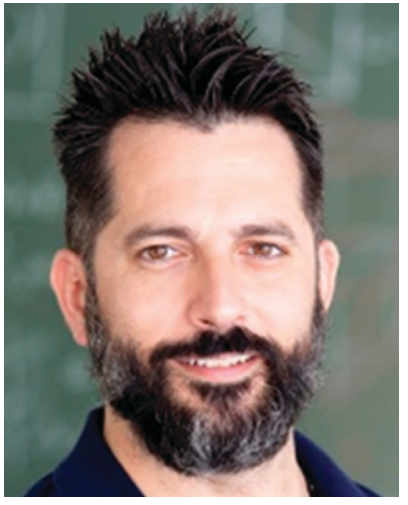

Iván Rivilla
Iván Rivilla graduated in Chemistry at the University of Castilla-La Mancha. He obtained his $P h D$ degree in Chemical Sciences in 2008. He did his first post-doctoral internship under the supervision of Prof. P. J. Pérez at the University of Huelva in 2009. In 2012, he moved to the University of the Basque Country (UPV/ EHU). Since 2018, he has also been a visiting researcher at the La Sapienza University of Rome.

Since 2020 he has been an Ikerbasque Research Associate at the Donostia International Physics Center (DIPC). His main research areas range from cycloaddition reactions to the design and synthesis of new chemical entities with biological activity. In addition, he is interested in the design and synthesis of new chemical sensors, for applications in particle physics or in the detection of pathogens. More recently, he has also begun to study reaction mechanisms by means of DFT calculations. 
sensing of cations is very well-suited for the detection of special events such as neutrinoless double beta decay nuclear processes. $^{5}$

Different techniques developed so far for the detection of metallic ions include atomic absorption spectroscopy, voltammetry, electrochemical analysis, inductively coupled plasma spectrometry, laser induced breakdown spectroscopy, neutron activation analysis and UV-Vis spectroscopy, among others. ${ }^{6,7}$ However, fluorescence ${ }^{8}$ is perhaps the most frequently used because of the relative simplicity of the associated devices, its very high sensitivity and its excellent spatial and temporal

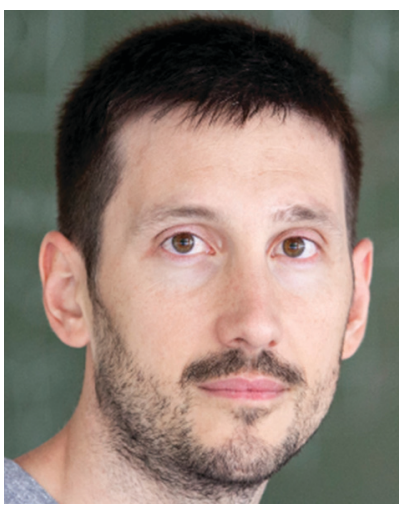

Francesc Monrabal
Francesc Monrabal graduated in Physics at the University of Valencia. He obtained his PhD at the same University developing the technology of gaseous xenon TPC for double beta decay search. In 2015 he started working with Prof. David R. Nygren at the University of Texas at Arlington where he was in charge of the design and construction of the Time Projection Chamber (TPC) for the NEXT (Neutrio Experiment with a Xenon Time Chamber)-White detector becoming a technical coordinator of the NEXT Collaboration the same year. Since 2019 he has been an Ikerbasque Research Fellow at the Donostia International Physics Center (DIPC), where he is leading the effort of the construction of the NEXT-100 detector. resolution. $^{9-15}$ Since in many samples different metal cations can coexist, the corresponding sensors must exhibit high selectivity. In this respect, fluorescence sensing arrays have been developed as a useful alternative based on ensembles of different less selective sensors that exhibit characteristic responses for different analytes. ${ }^{16}$

Many different types of fluorescent sensors able to detect metal cations have been described, including nanoparticles, ${ }^{17}$ carbon-based quantum dots, ${ }^{18,19}$ and dendrimers, ${ }^{20}$ as well as metal-organic frameworks (MOFs), ${ }^{21}$ and biomolecules such as DNA $^{22}$ and proteins. ${ }^{23}$ These clustered and macromolecular sensors are not included in the present Perspective Article, which is focused on discrete fluorescent molecular sensors.

The fluorescence emission ${ }^{24}$ spectrum of a given fluorophore is produced after excitation at a certain wavelength (normally in the UV region), followed by emission of photons at larger wavelengths. The Stokes shift expresses the difference between the maximum absorption wavelength $\lambda_{\mathrm{abs}}^{\max }$ and the maximum emitted wavelength $\lambda_{\mathrm{em}}^{\max }$. If this difference is expressed in wavenumbers, the corresponding $\Delta \bar{\nu}_{\text {Stokes }}$ is given by

$$
\Delta \bar{\nu}_{\text {Stokes }}=\frac{1}{\lambda_{\mathrm{abs}}^{\max }}-\frac{1}{\lambda_{\mathrm{em}}^{\max }}
$$

Since $\lambda_{\mathrm{em}}^{\max }>\lambda_{\mathrm{abs}}^{\max }, \Delta \bar{\nu}_{\text {Stokes }}>0$. The excitation process generates a transition from the ground state $S_{0}$ of molecule A to the first singlet excited state $S_{1}\left(A^{*}\right)$. The decay from $S_{1}\left(A^{*}\right)$ to $\mathrm{S}_{0}(\mathrm{~A})$ is determined by the radiative kinetic constant $k_{\mathrm{r}}^{S}$

$$
\mathrm{A} * \stackrel{k_{\mathrm{r}}^{S}}{\rightarrow} \mathrm{A}+\operatorname{photon}\left(\lambda_{\mathrm{em}}\right)
$$

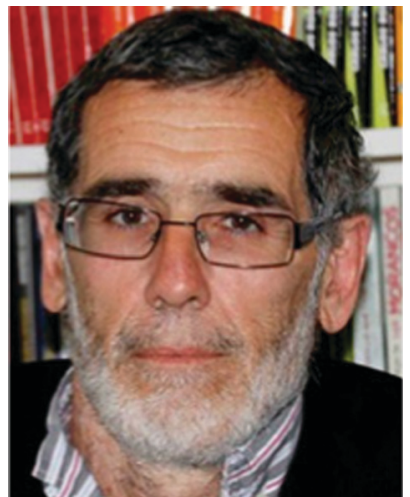

Juan J. Gómez-Cadenas
Juan José Gómez Cadenas received both his $M S c$ degree and his $P h D$ from the University of Valencia. He has been a Staff member at the European Laboratory for Particle physics (CERN), a full professor at the University of Valencia and a full professor at CSIC. Since 2017, he has been an Ikerbasque Professor at DIPC. He is the cospokesperson of NEXT, a leading experiment searching for neutrinoless double beta decay processes $(\beta \beta O \nu)$. Together with the other authors of this paper and the members of the NEXT Collaboration, he is leading an interdisciplinary effort to develop a background-free $\beta \beta O \nu$ experiment in xenon based on the tagging of the xenon daughter $\left(\mathrm{Ba}^{2+}\right)$ in the double beta decay through the use of molecular sensors.

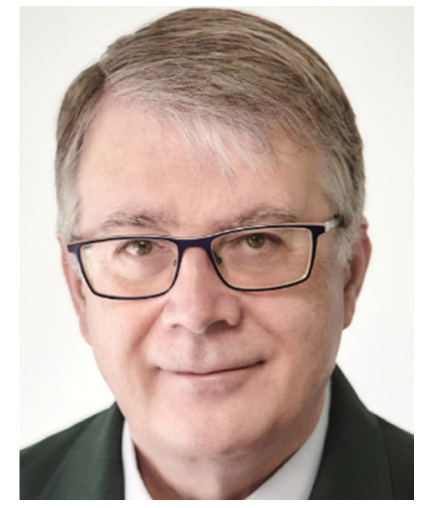

Fernando P. Cossío
Fernando P. Cossio received his MSc degree in chemistry from the University of Zaragoza and his PhD in organic chemistry from the University of the Basque Country (UPV/EHU). Since 2002, he has been a full professor in the $U P V / E H U$, where he served as the Dean of the Faculty of Chemistry and the vice chancellor for research and international relations. Since 2009, he has been the scientific director of Ikerbasque, the Basque foundation for the advance of science. His research interests are focused on experimental and computational aspects of cycloaddition chemistry and $\mathrm{C}-\mathrm{C}$ bond forming reactions, with special emphasis in the design and chemical synthesis of new molecules with useful biological and photophysical properties in medicinal chemistry, molecular imaging and neutrinoless double beta decay reactions leading to $\mathrm{Ba}^{2+}$ cations. 
and follows a usual first-order kinetics in the form

$$
\left[{ }^{1} \mathrm{~A} *\right]_{t}=\left[{ }^{1} \mathrm{~A} *\right]_{0} \exp \left(-\frac{t}{\tau_{\mathrm{S}}}\right)
$$

In the latter equation, $\left[{ }^{1} \mathrm{~A}^{*}\right]_{0}$ is the concentration of excited molecules at $t=0$, which corresponds to the instant of the pulse light excitation, and $\tau_{\mathrm{S}}$ is the lifetime of the excited state $S_{1}$ :

$$
\tau_{\mathrm{S}}=\frac{1}{k_{\mathrm{r}}^{S}+k_{\mathrm{nr}}^{s}}
$$

where $k_{\mathrm{nr}}^{S}$ is the total kinetic constant associated with nonradiative processes that do not result in fluorescence emission.

The time-dependent intensity of the fluorescence emission $i_{\mathrm{F}}(t)$ after excitation at $t=0$ is proportional to the concentration of molecule $\mathrm{A}$ in the singlet excited state and to the kinetic constant associated with the fluorescence emission:

$$
i_{\mathrm{F}}(t)=k_{\mathrm{r}}^{S}\left[{ }^{1} \mathrm{~A}^{*}\right]_{t}=k_{\mathrm{r}}^{S}\left[{ }^{1} \mathrm{~A}^{*}\right]_{0} \exp \left(-\frac{t}{\tau_{\mathrm{S}}}\right)
$$

It is important to note that the measured fluorescence intensity $I_{\mathrm{F}}$ is obtained from the total decay at different wavelengths and is expressed in arbitrary units (a.u., not to be confused with atomic units) that depend on the experimental conditions.

The fluorescence quantum yield for a given $\mathrm{A}^{*} \rightarrow$ A transition is the fraction of excited molecules $S_{1}\left(A^{*}\right)$ that decay to the ground state $\mathrm{S}_{0}(\mathrm{~A})$ with emission of photons (vide supra):

$$
\phi_{\mathrm{F}}=k_{\mathrm{r}}^{S} \tau_{\mathrm{S}}=\frac{k_{\mathrm{r}}^{S}}{k_{\mathrm{r}}^{S}+k_{\mathrm{nr}}^{S}}
$$

Thus, the quantum yield gives the fraction of emitted photons during the total decay with respect to the absorbed photons. This magnitude gives crucial information to measure the efficiency of fluorescence emission. Another relevant parameter is the brightness ${ }^{4,23}$ of the fluorescence emission $B_{\mathrm{F}}$, which is the product of the quantum yield and the molar extinction coefficient $\varepsilon_{\text {abs }}$ at the excitation light wavelength.

$$
B_{\mathrm{F}}=\phi_{\mathrm{F}} \varepsilon_{\mathrm{abs}}
$$

All these combined magnitudes characterize the photophysical behaviour of a given fluorophore.

The key point in fluorescence sensing of different metallic cations is the change of these magnitudes upon coordination or binding of the sensor with the analyte. In a fluorescent monocolour sensor the emitted photons are of identical wavelengths $\lambda_{\mathrm{em}}$ in the free state and upon coordination or binding with the metal cation. This can occur via photoinduced electron transfer $^{1,25}$ (PET) phenomena via an electronic donation from one heteroatom of the sensor that quenches the fluorescence relaxation of the fluorophore in the free $S_{1}$ state. Upon coordination, this donation is directed to the metal and the fluorescence decay takes place efficiently thus resulting in an off-on transition (turn-on enhancement) on going from the free to the metalcoordinated state. Another less frequently observed PET consists of the reverse on-off process (turn-off quenching). In most cases, the PET perturbations of the relaxation processes result in preferential emission of photons with $\lambda_{\mathrm{em}}$ values similar for free and bound states, the only difference being the very low and very high intensities in the off and on states, respectively. Therefore, the larger the difference between the intensities of the off and on states, the higher the sensitivity of the sensor. These monocolor probes require very high intensitybased separation factors between their 'off' and 'on' states; such separation factors are challenging to attain, given various analyte-independent factors which affect the probe intensity (e.g. fluctuations in the excitation source, the effect of the microenvironment, variations in the probe concentration, etc.). ${ }^{26,27}$ In this Perspective Article we rather focus on bicolor probes which rely on wavelength separation and are not affected by such factors.

Molecular bicolour sensors emit photons with different wavelengths in the free $\left(\lambda_{\mathrm{em}}\right)$ and bound $\left(\lambda_{\mathrm{em}}^{\prime}\right)$ states. They can also be described as dual emission ${ }^{28}$ ratiometric probes with signal changes associated with two independent or correlated emitters. ${ }^{27}$ Depending on the magnitude of $\lambda_{\mathrm{em}}^{\prime}$ with respect to $\lambda_{\mathrm{em}}$, blue (hypsochromic) or red (bathochromic) shifts will be observed. Thus, both free and bound bicolour sensors can be active fluorescent emitters within an on-on' scheme. In this case, the sensitivity will be determined by the respective intensities and the corresponding photophysical parameters. Two relevant parameters are the wavelength difference $\Delta \lambda_{\mathrm{F}}=\lambda_{\mathrm{em}}^{\prime}-\lambda_{\mathrm{em}}$ and the peak discrimination factor $f_{\lambda}$, defined as

$$
f_{\lambda}=\frac{I_{\lambda}^{\prime}-I_{\lambda}}{I_{\lambda}}
$$

where $I_{\lambda}^{\prime}$ and $I_{\lambda}$ are the intensities of the signals characterized by a wavelength $\lambda$ at the bound and free states, respectively. This factor is an indicator of the expected signal cleaning of the metal-bound sensor with respect to its free unbound state, especially when the concentration of the metallic cation is very low (vide infra). Thus, the greater the separation dictated by $f_{\lambda}$, the lesser the overlap between both signals. Aside the intrinsic values of quantum yield, brightness and intensity, these last two factors will determine the sensitivity of the molecular sensor with respect to the background. This is particularly important in cation-tagging experiments with extremely low concentration of the metallic cation (eventually, only one!), in which the concentration of the free molecular bicolour sensor is therefore orders of magnitude more higher than that of the bound sensor.

Another important aspect associated with the design of molecular fluorescent bicolour sensors is the necessity of developing sensors able to capture cations in the gas phase. This in turn implies the exploration of supramolecular chemistry on solid-gas interfaces, a research field much less 
developed than its in-solution analogues. The use of single cation identification has been proposed as a way to dramatically reduce the radioactive background ${ }^{29}$ in xenon detectors looking for neutrinoless double beta decay. The ${ }^{136} \mathrm{Xe}$ isotope decays into barium, emitting two electrons (and two anti-neutrinos in the standard process). Identification of a coincidence signal of the two electrons with the appropriate energy with the appearance in the active volume of $\mathrm{B}^{2+}$ ion will be an unequivocal signature of the neutrinoless process and, consequently, a demonstration that neutrinos are their own anti-particles (Majorana ${ }^{30}$ particles). Demonstrating this hypothesis would have deep consequences in particle physics and in cosmology. ${ }^{31,32}$ A promising "wet" barium tagging experiment has been reported as a proof of concept. $^{33}$ In order to reproduce the required "dry" conditions imposed by these experiments, several interesting barium-tagging sensors have been reported so far, ${ }^{34,35}$ all of them based on PET off-on fluorescence emission. Our approach to barium-tagging is instead based on the development of suitable on-on' bicolour fluorescent sensors, which benefit from a wavelength-shifting mechanism.

The many contributions to the development of ratiometric molecular fluorescent sensors for cations have been comprehensively described in leading reviews and book chapters in the area. ${ }^{9,26,27,36-42}$ In this Perspective Article, we shall present and discuss the main features and design criteria of molecular bicolour fluorescent sensors for metallic cations, intended for the detection of extremely low cation concentrations (eventually only one) on a surface covered by unbounded sensor molecules, in dry media. In the following sections, we will describe the main modes of sensor-cation interactions and the electronic phenomena responsible for the change in the fluorescence spectra upon metal coordination or after interaction with the cationic centre. We will also report on recent results obtained in our laboratories on barium tagging in dry media involving solid-gas phases.

Bicolour fluorescent probes can be considered a special type of the so-called single-fluorophore ratiometric probes with two analyte-sensitive emission signals, as they change the emission intensity and wavelength upon interaction with the analyte. They can also be described as dual emission materials composed of two independent emitters (free and bound) in equilibrium. ${ }^{28,43}$ In general, these molecules incorporate different components that include a synthetic ionophore (the cation recognition-binding domain), the fluorophore and, optionally, a spacer and a linker to anchor the sensor with suitable surfaces or biomolecules. The covalent connections among these components can be different, thus giving rise to different sensor architectures, the most usual ones being those shown in Fig. 1A.

The binding groups (ionophores) are responsible for the efficient and preferably highly selective capture of the metal cation and can consist of chelating groups ${ }^{4}$ such as polycarboxylic acids B1, podands B2, coronands such as crown ethers ${ }^{\text {44-46 }}$ B3 and cryptands B4. Other ionophores ${ }^{47,48}$ such as calixarenes, cyclodextrins and pillararenes, not shown in Fig. 1B, have been described.

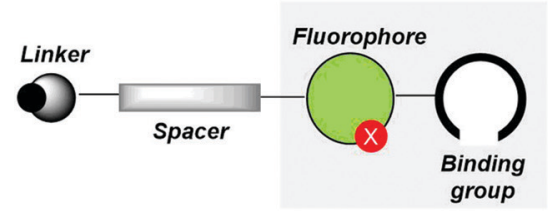

A
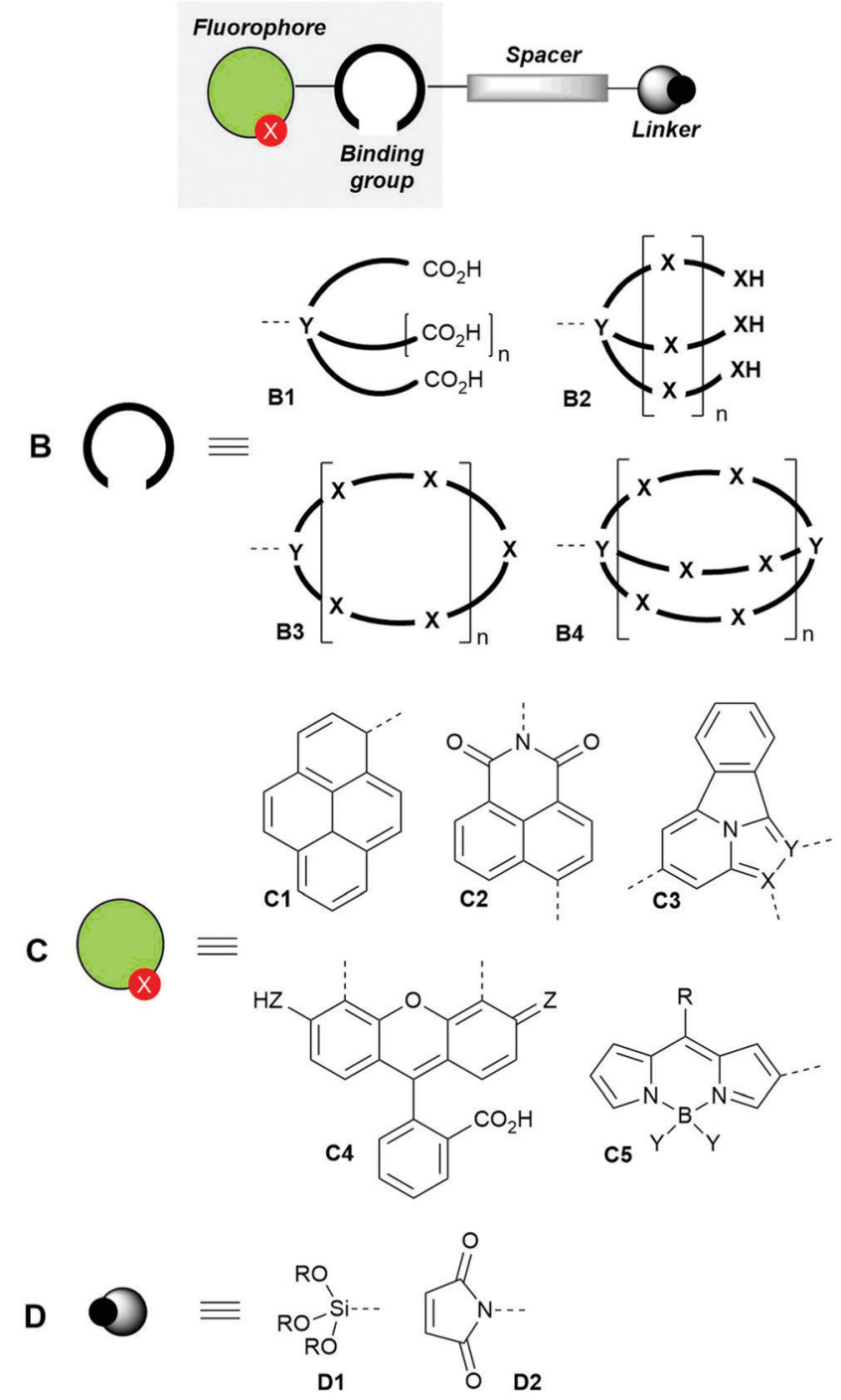

Fig. 1 General design patterns of fluorescent molecular sensors for cations (A), showing their main components: binding groups (ionophores, B), fluorophores (C) and linkers (D).

The structures of fluorophore components (Fig. 1C) are very varied $^{4}$ and comprise aromatic hydrocarbons such as pyrenes C1, and polyheterocyclic aromatic moieties such as naphthylimides C2, tetracyclic structures based on imidazo[1,2(5)a] pyridines $^{49,50} \mathbf{C 3}$, fluorescein derivatives $\mathbf{C 4}$ and BIODIPYbased structures C5, among others.

When required, the spacers can consist of aliphatic linear chains or polyethylene glycol (PEG) derivatives, which can intercalate with aromatic hydrocarbons or heterocycles. Finally, the linkers can be trialkoxysilyl derivatives D1 (very useful for anchoring the fluorescent molecular sensors to solid surfaces) or maleimides D2, which can bind - with low selectivity - cysteine-containing 


\section{A. Chelation-based bicolour sensors}

\section{A.1. Fluorophore + chelating group}

- Monomeric
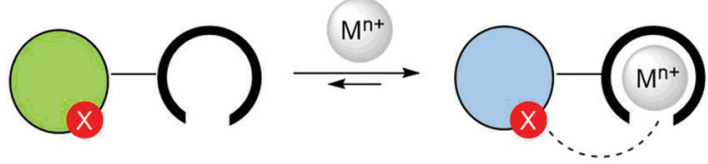

Dimeric
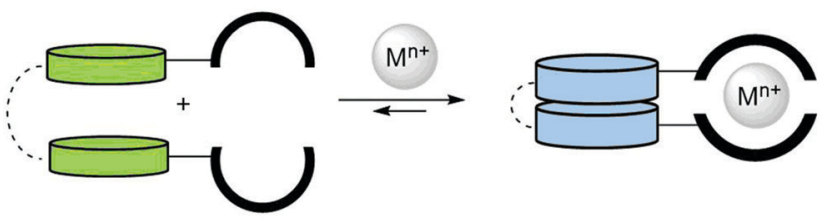

A.2. Chelating fluorophore
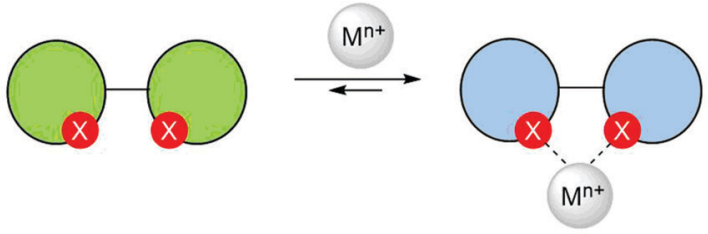

B. Reactivity-based bicolour sensors
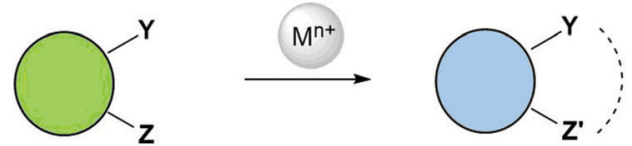

Fig. 2 Main types of bicolour sensors classified according to their structure and interaction with the metal. Different chelation modes (A, monomeric and dimeric chelating groups, as well as chelating fluorophores) are shown. Bicolour sensors in which the change in the fluorophore is generated by a chemical reaction with the metallic ion are also shown (mode B). Optional spacer and linker components are omitted.

proteins or biothiols, thus giving rise to the corresponding conjugated derivatives. ${ }^{51}$

Molecular fluorescent bicolour sensors can change the intensity and emission wavelength of their fluorescence signals in different ways after interacting with metal cations (Fig. 2). When an ionophore is present, metal binding can modify the photophysical properties of the sensor via formation of monomeric and dimeric complexes (Fig. 2, mode A.1). In some cases, when the fluorophore itself is able to bind directly the metallic cation, the photophysical properties of the chelate can be different to those of the free fluorophore (Fig. 2, mode A.2), thus yielding a bicolour sensor. In many cases, a heteroatom $\mathrm{X}$ possessing a suitable lonepair present in the fluorophore can also bind the metallic centre thus forming a complex in which both the synthetic ionophore and the heteroatom are bound to the cationic analyte (Fig. 2, note the dashed line in monomeric complex A.1). Finally, reactive functional groups decorating the fluorophore can react selectively with the metal to be transformed into a different fluorophore, thus giving rise to an ionophore-free fluorescent bicolour sensor (Fig. 2B). These considerations apply to not only single emitters from one excited electronic state, but also to dual emitters with a sole fluorophore possessing two emitting states and dual emitters with two independent or correlated emission centres. ${ }^{28}$

\section{Mechanisms of bicolour sensing}

\subsection{Intramolecular charge transfer (ICT)}

If changes in the electronic structure of the fluorescent bicolour sensor are considered, one possibility consists of modifying the polarization patterns between substituents of the fluorophore in the free and bound states. This process is named Photoinduced Charge Transfer (PCT), which almost in all cases takes place intramolecularly. Therefore, the latter process will be referred to as Intramolecular Charge Transfer (ICT). In these systems, an electron-releasing group (ERG), which acts as a donating group, transfers a certain electron density to an electron-withdrawing group (EWG), which is the acceptor of this charge transfer. This process involves complex electronic interactions. Within a simplified scheme involving delocalized frontier molecular orbitals, two situations can be distinguished, depending on the proximity of ERG and EWG moieties to the metallic cation. Of course, specific electronic interactions can be important, especially when there is a direct union between the fluorophore and the metal ion. When the ERG is close to the $\mathrm{M}^{n+}$ centre bound to the ionophore, the two-electron ERG $\rightarrow \mathrm{M}^{n+}$ interaction will compete with the ERG $\rightarrow$ EWG donation thus increasing the $\nu_{\mathrm{em}}^{\prime}$ frequency associated with the fluorescence $\mathrm{S}_{1} \rightarrow \mathrm{S}_{0}$ transition (Fig. 3A). Therefore, a blue shift will be observed, in many cases with a certain decrease in brightness. Alternatively, when the EWG is closer to the cation, the ERG $\rightarrow$ EWG donation will increase in magnitude and the EWG-M ${ }^{n+}$ interaction will result in the lowering of the lowest unoccupied molecular orbital (LUMO) energy of the fluorophore, thus resulting in a lower $\nu_{\text {em }}^{\prime}$ frequency and the corresponding red shift, usually associated with a brighter signal (Fig. 3B). In both cases, the sensitivity of the ICT sensor correlates with the respective differences in brightness, as well as with the peak discrimination factors (vide supra).

\subsection{Excimers}

Another way to obtain radiometric bicolour sensors consists of designing fluorophores that, upon binding the metallic cation through the adequate ionophore, form dimers (homo- or heterodimers) that are more stable in the $\mathrm{S}_{1}$ excited state. Thus, an excimer (excited dimer) originates from the interaction of an excited fluorophore with a nearby congener in the ground state. If the two interacting units are non-identical, the whole chemical entity is named an exciplex (excited complex). The two units can be connected by a spacer and incorporate their respective incomplete ionophore components that, upon coordination with the metallic cation, generate the optimal binding group (Fig. 4A). An analysis of the frontier molecular orbitals (FMOs) of the monomeric units of the sensor at the $\mathrm{S}_{0}$ and $\mathrm{S}_{1}$ (Fig. 4B) states shows that the energy levels of the FMOs of the excimer (or the exciplex) are modified upon dimerization 

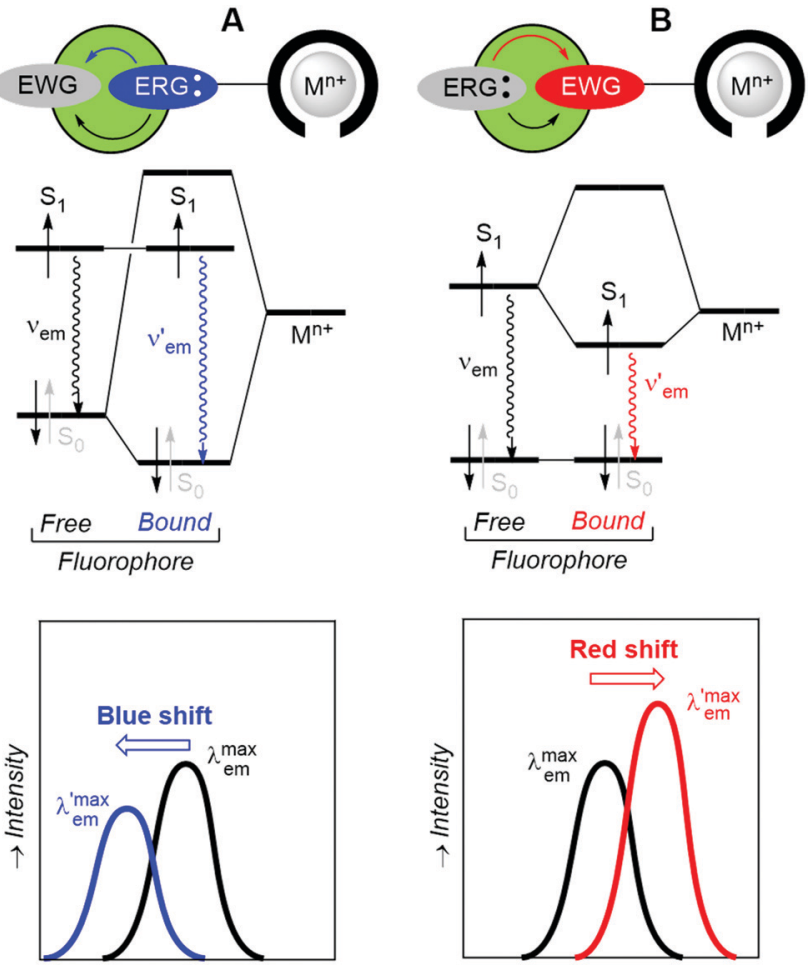

Fig. 3 Schematic representation of intramolecular charge transfer processes (ICT) that modify the fluorescence emission of bicolour sensors in the presence of cations $M^{n+}$. (A) Blue shift effect of an electron-releasing group (ERC) close to the ionophore. (B) Red shift effect of an electronwithdrawing group (EWG) close to the ionophore.

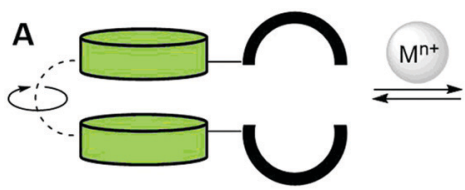

B

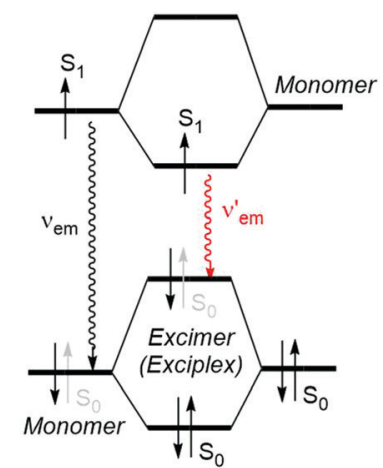

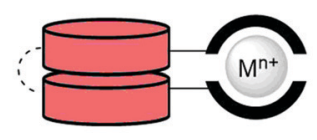

C

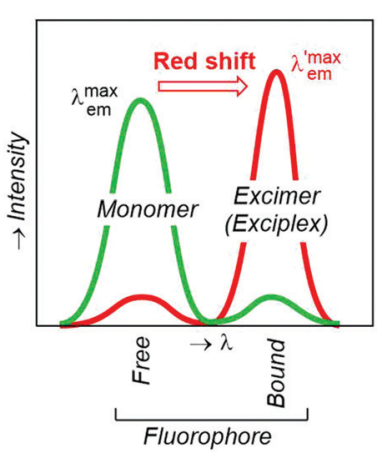

Fig. 4 (A) Cartoon showing the formation of excimers/exciplexes in the presence of a metallic cation. (B) Qualitative diagram showing the distortion of the FMO energy levels in the $S_{1}$ state of the excimer with respect to those of the monomer. (C) Red shift of the emission spectra of monomers and excimers/exciplexes associated with the diagram shown in (B).

and usually result in a shorter HOMO-LUMO gap (HOMO stands for the highest occupied molecular orbital). ${ }^{52}$ In turn, this shrinkage in energy levels generates a red shift that permits to discriminate the free and bound states of the sensor. The binding ability of the partial ionophores in the free monomeric state and the stability of the excimer/exciplex can result in a background signal that, depending upon the respective intensities, permits to establish an accurate measurement of the radiometric response (Fig. 4C). In other cases, the excimer/ exciplex is relevant in the absence of the metallic cation, whose coordination results in the total or partial loss of fluorescent response associated with excimer inhibition promoted by cation coordination.

\subsection{FRET}

A related phenomenon in which the emission involves an interaction between two fluorophores (Fig. 5A) is the Förster resonance energy transfer (FRET). ${ }^{53-55}$ This process consists of a non-radiative excitation transfer ${ }^{56}$ from the excited donating $\left(\mathrm{D}^{*}\right)$ group to the acceptor group (A) in its ground state (Fig. 5B). The latter group relaxes via an $\mathrm{A}^{*}\left(\mathrm{~S}_{1}\right) \rightarrow \mathrm{A}\left(\mathrm{S}_{0}\right)$ process thus emitting with a different wavelength (Fig. 5C). ${ }^{57}$ Any FRET requires appropriate relative orbital energies associated with the FMOs of D and A. In the case of ratiometric bicolour sensors, the FRET is usually observed when the sensor is bound to the cationic centre. It is noteworthy that the distance $R$ required for FRET is significantly larger than the average values of the combined van der Waals radii of the $\mathrm{D}$ and $\mathrm{A}$ groups. The Förster distance $R_{0}$ is defined as the distance at which the FRET efficiency $\phi_{\text {FRET }}=\left[1+\left(R / R_{0}\right)^{6}\right]^{1-}$ is 0.5 . For instance, if $\mathrm{D}=$ pyrene and A = coumarin, $R_{0}=3.9 \mathrm{~nm} .^{58}$ The FRET efficiency also depends on the relative orientation of $\mathrm{D}$ and $\mathrm{A}$, as well as
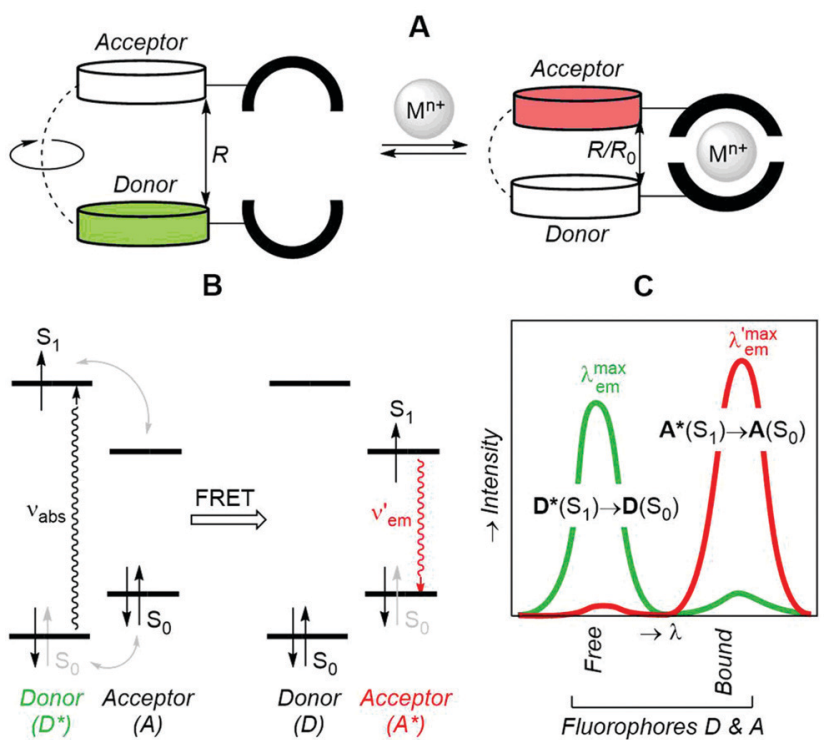

Fig. 5 (A) Schematic representation of a fluorescent sensor incorporating donor and acceptor units. $R_{0}$ is the Förster radius (see the text). (B) Försterresonance electron transfer (FRET) associated with the frontier molecular orbitals of the donor and the acceptor. (C) A representation of the fluorescence emission spectra of the FRET sensor in the free and bound states. 
on the overlap between the emission spectrum of $\mathrm{D}$ and the absorption spectrum of A.

\subsection{Two-component fluorophores}

In the previous sections, we have presented bicolour sensors based on fluorophores that act as indivisible photoactive ensembles. Another possibility consists of splitting a given fluorophore into two separate rotatable units connected by a single covalent bond (Fig. 6). In turn, this two-unit fluorophore 1 is covalently attached to an ionophore. When a metallic cation is captured by the latter binding moiety, the nature of the interaction is reinforced by additional coordination with a heteroatom of fluorophore $\mathbf{1}$, thus yielding an ICT-complex (Fig. 2, A.1 pattern) that in addition incorporates a $\pi$-cation interaction ${ }^{59}$ between the metallic centre $\mathbf{M}^{n+}$ and the $\mathrm{Ar}_{2}$ group gathered in Fig. 25. This $\mathrm{Ar}_{2}-\mathbf{M}^{n+}$ complex promotes a $\mathrm{Ar}_{2} \rightarrow \mathrm{M}^{n+}$ charge transfer, as well as a rotation of $\mathrm{Ar}_{2}$ with respect to the polyheterocyclic $\mathrm{HetAr}_{1}$ unit. The outcome of these metal-induced changes is the decoupling between the HetAr and $\mathrm{Ar}_{2}$ groups. Therefore, the new $\mathrm{HetAr}_{1}$ fluorophore 2 thus formed (Fig. 25) is less conjugated than its predecessor fluorophore 1 in the unbound state and will emit photons with shorter wavelength. If an optical filter is installed in the detection system to hide the emission associated with fluorophore $\mathbf{1}$, this blue shift should permit an essentially background-free signal upon cation complexation if the peak discrimination factor $f_{\lambda}$ is large enough.

\section{Design and validation of bicolour probes}

All of the above discussed bicolour sensors have been classified in terms of the chemical interactions involved

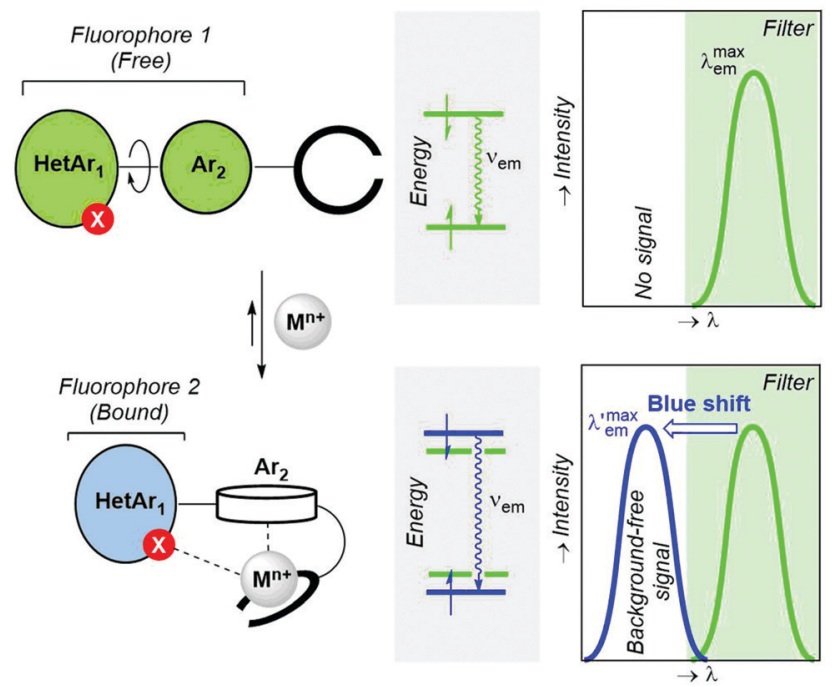

Fig. 6 Design criteria of two-component fluorescent bicolour indicators (FBI) based on the decoupling of one component of the starting fluorophore upon metal coordination. The metal-induced hypsochromic shift and the recording of a background-free signal by means of an optical filter are also highlighted. HetAr and Ar denote polyheterocyclic aromatic and aromatic groups, respectively. (fluorophore-ionophore, direct chelation by the fluorophore and a chemical reaction with the cation) or the electronic effects that promote the change in the wavelength of the emitted photons (ICT, excimer/exciplex, FRET and related phenomena). In the following sections, an overview of selected examples of these strategies for the construction of bicolour fluorescent probes for cations is presented and discussed.

\subsection{ICT-Based sensors}

Many review articles and books describe the photo-induced ICT phenomenon and the potential applications of ICT-based compounds. ${ }^{24,60}$ In polar solvents, rearrangement of the solvation shell around the ICT excited state produces its stabilization and the concomitant destabilization of the landing ground state, raising its energy level. ${ }^{41}$ Therefore, these compounds may show dual emission: a peak in the most energetic region of the emission spectra that originates at the locally excited state (LE), and a red-shifted signal from the solvent-stabilized ICT excited state. Additionally, if the donor and the acceptor are connected through a rotating $\pi$-electron bridge, a relaxation of the ICT excited state can occur through an intramolecular rotation, decoupling the orbitals of the donor and acceptor, forming a highly polar excited state. This phenomenon is referred to as "twisted intramolecular charge transfer" (TICT), and it is characterized by extra-large Stokes shifts in polar solvents.

Both ICT- and TICT-based fluorophores are suitable scaffolds for the construction of bicolour probes for cations by integrating a cation receptor either on the ERG or on the EWG sites of the fluorophore (Fig. 3). The simplicity of the design makes ICT-based sensors the most widely used ratiometric probes for cations. Among the earliest examples are Indo-1 and Fura-2 (Fig. 7), developed in 1985 by Tsien and collaborators. ${ }^{61}$ Nowadays, Indo-1 and Fura-2 are the leading compounds of a series of commercially available fluorescent ratiometric probes for $\mathrm{Ca}^{2+}$ and other cations. ${ }^{62}$ They combine a stilbene skeletal fluorophore (reinforced by extra heterocyclic bonds) with the well-established BAPTA fragment as an ionrecognition site. These molecular probes are characterized by a strong fluorescent ratiometric response to $\mathrm{Ca}^{2+}$. Because of the inhibition of the ICT upon coordination of the $\mathrm{Ca}^{2+}$ to the
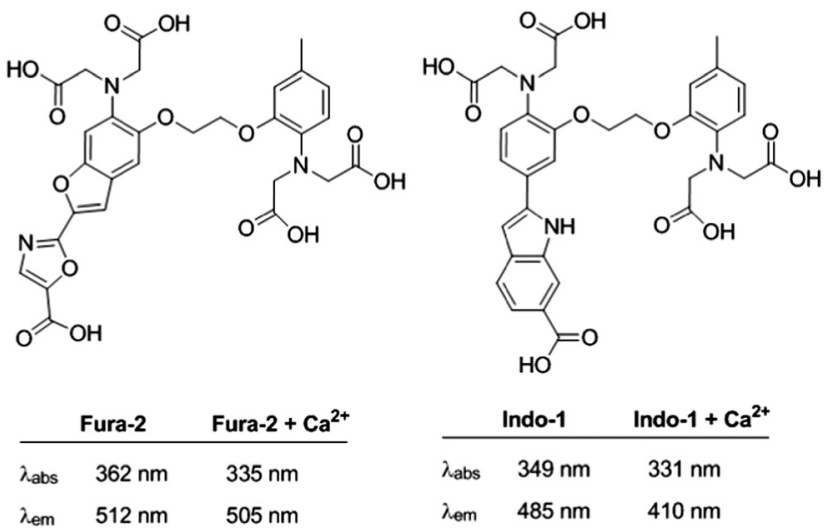

Fig. 7 Fura-2 and Indo-1 ICT-based sensors for $\mathrm{Ca}^{2+}$ 
aniline nitrogen of the BAPTA receptor, a blue-shifted fluorescenc emission is observed (Fig. 3A). One of the main limitations of these probes was the need for UV-light excitation. It was circumvented using two-photon absorption (TPA) microscopy, which permitted the study of cation distribution in live tissues. ${ }^{63}$

More recent ICT-based sensors are based on fluorophores that can be excited in the visible region. In 2019 Woodruff and O'Halloran synthesized a family of BODIPY-based fluorophores containing an appended $N, N, N^{\prime}$-tris(2-pyridylmethyl)-1,2ethanediamine (trispicen) chelator as a $\mathrm{Zn}^{2+}$ receptor site. ${ }^{64}$ Among them, ZincBY-4 (Fig. 8), containing a pending acetamide on the 5-position of the BODIPY core outperformed other derivatives due to intramolecular hydrogen bonding between the amide hydrogen and the fluorine atoms of the $\mathrm{BF}_{2}$ centre, thus increasing the $\mathrm{Zn}^{2+}$ affinity. In polar solvents, the ICT-based fluorescence of the free probe is strongly quenched, whereas a blue-shifted emission is recovered upon $\mathrm{Zn}^{2+}$ chelation due to disruption of the ICT process.

In 2017, Han, Zang and colleagues published ratiometric probes for $\mathrm{Cd}^{2+}$, also based on cation-induced ICT depletion, characterized by a long wavelength fluorescence emission $\left(\mathrm{Me}_{4}\right.$ BOPHY-BPA in Fig. 8). ${ }^{65}$ The sensor consisted of a tetramethylated BOPHY fluorophore conjugated to bis(pyridine-2-yl) amine (BPA) podand acting as a $\mathrm{Cd}^{2+}$ selective receptor. $\mathrm{Cd}^{2+}$ Chelation caused a clear hypsochromic shift of the fluorescence emission revealing the characteristic yellow emission of the $\mathbf{M e}_{4}$ BOPHY dye. In 2019, Zeng et al. pushed this concept further developing a $\mathrm{Hg}^{2+}$-probe (L in Fig. 8) with NIR emission in both the holo and the apo form. ${ }^{66}$ This sensor consists of a chromenylium-cyanine dye containing a conjugated $\mathrm{NS}_{2} \mathrm{O}_{2} \mathrm{Hg}^{2+}$-chelating unit. The strong ICT process between the $\mathrm{N}$-atom of the cation receptor and the fluorescent dye, together with the NIR emission of the naked fluorophore, permitted the authors to construct this ratiometric NIR sensor operative under visiblelight excitation.

The clear advantages of TPA microscopy compared to conventional one-photon absorption (OPA) microscopy for the analysis of cation distribution in live cells, tissues, and organisms (i.e. improved penetration, reduced toxicity, reduced autofluorescence, and minimal light scattering) drove the development of molecular probes optimized for TPA. ${ }^{67-69}$ When considering ICT-based sensors, this has been pursued by binding the cation recognition site to the acceptor domain of an ICT-fluorophore. Therefore, upon metal binding, rather than ICT depletion (as observed in the examples mentioned above) the polarization of the ICT excited state will increase. This probe configuration should not only produce a bathochromic shift in the fluorescence emission of the cation-bound probe (Fig. 3B), but also enhance the TPA cross-section, and the brightness of the fluorescence emission. ${ }^{69}$

Based on this premise, in the last few years a series of ratiometric TPA probes appeared in the literature. Several $\mathrm{Zn}^{2+}$ selective sensors have been developed based on a BPA podand bound to different fluorophores. Representative examples are shown in Fig. 9. ${ }^{70-73}$ MPVQ-series, initially developed by Meng's
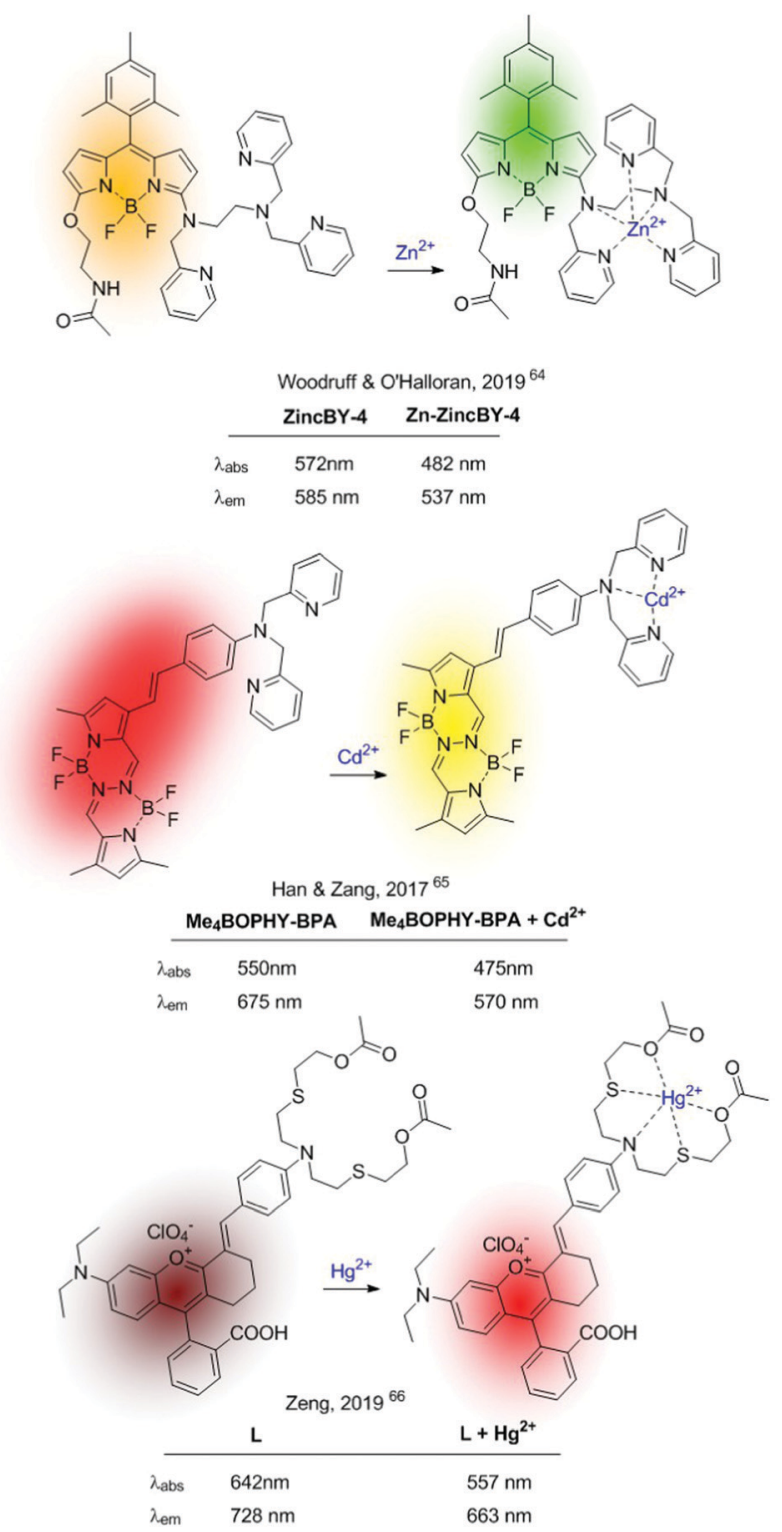

Fig. 8 Recent examples of metal-induced ICT depletion probes.

group ${ }^{73}$ consisted of BPA-appended quinoline as the fluorescent skeleton with a conjugated donor on the 6-position, whereas chromis-1 contains 2-arylthiazole as a push-pull fluorophore. In all cases, a red-shifted fluorescence was observed in the presence of $\mathrm{Zn}^{2+}$, which was accompanied by an increased TPA cross-section.

More complex ratiometric sensors for cations, optimized for TPA, have been constructed using centrosymmetric donor- $\pi-$ acceptor- $\pi$-donor quadrupolar configurations, in which the cation recognition site is integrated into the central acceptor unit, thus constituting a chelating fluorophore (Fig. 2, pattern A.2). The GBC probe (Fig. 10), developed in $2014,{ }^{74}$ was a ground-breaking example of sensors based on this structural motif and inspired further developments. ${ }^{75,76}$ The cation recognition is achieved through a central bipyridine unit with two appended carbazole donors linked through vinylic $\pi$-electron 

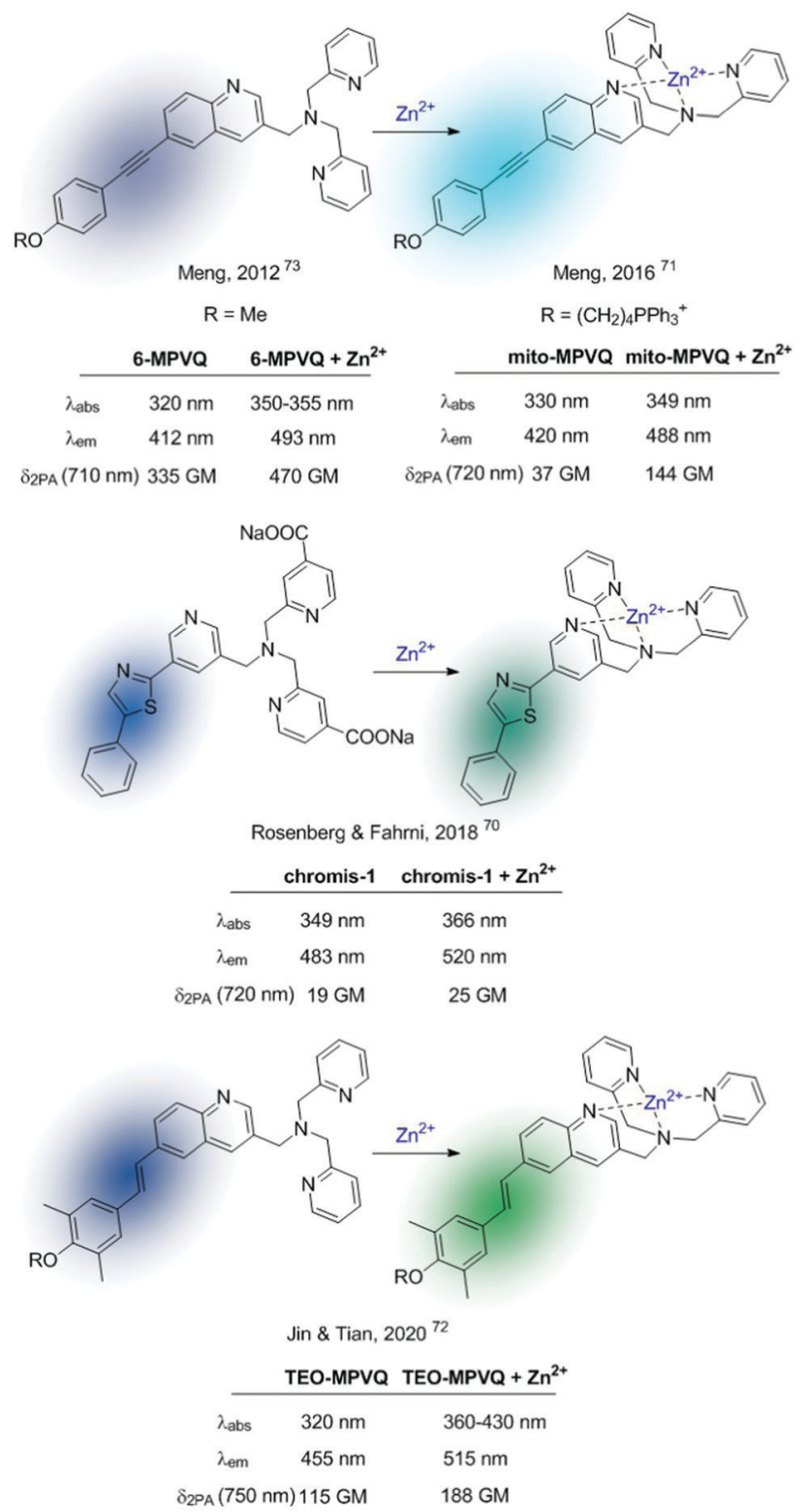

Fig. 9 TPA ICT-based ratiometric probes using BPA as a selective $\mathrm{Zn}^{2+}$ receptor.

bridges. GBC also contains oxyethylene side chains to increase its solubility in aqueous media and its cell permeability. The emission red shift and the enhanced TPA observed upon metal binding were initially attributed to ICT, because of the increased electron-withdrawing character of the $\mathrm{Zn}$-coordinated bipyridine unit. The mechanism responsible for the large TPA characteristic of these systems has been further analysed from a theoretical perspective aiming at establishing structure-property relationships. ${ }^{77-79}$ These studies revealed that fluorescence emission under two-photon excitation originates from the $\mathrm{S}_{2}$ excited state rather than from $\mathrm{S}_{1}$. Additionally, coordination of the $\mathrm{Zn}^{2+}$ cation requires the rotation of the central bipyridine unit from the trans to the cis coplanar form. This conformational change results in an increase of the dipole and quadrupole moments of
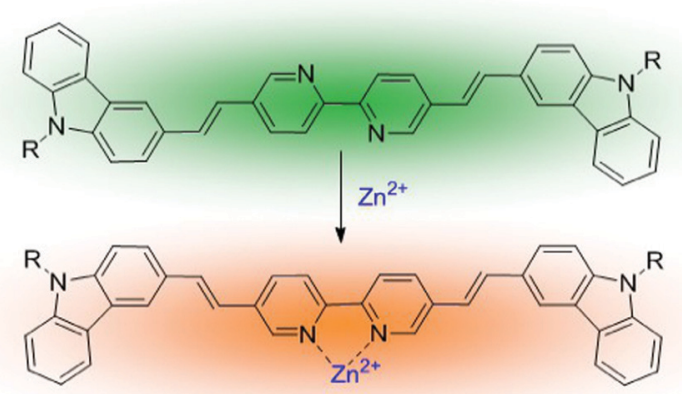

$$
\begin{aligned}
& \text { Zhao \& Ajayaghosh, } 2014^{74} \\
& \text { GBC } \mathrm{GBC}+\mathrm{Zn}^{2+} \\
& \lambda_{\text {abs }} \quad 403 \mathrm{~nm} \quad 430 \mathrm{~nm} \\
& \lambda_{\mathrm{em}} \quad 530 \mathrm{~nm} \quad 610 \mathrm{~nm} \\
& \delta_{2 \mathrm{PA}}(820 \mathrm{~nm}) 107 \mathrm{GM} \quad 1433 \mathrm{GM}
\end{aligned}
$$

Fig. $10 \mathrm{GBC}$ ratiometric probe for $\mathrm{Zn}^{2+}$.

the molecule by a loss of the centre of inversion present in the most stable conformation of the unbound probe. ${ }^{77}$

3.1.1. Multi-target sensors. The electronic origin of the shift in the emission observed in ICT-based sensors makes it feasible to develop multi-target sensors, able to discriminate between similar cations due to the different emission colours of their corresponding complexes. The cation-dependent polychromic emission observed in some ICT-based sensors has been attributed to differences in electronic affinities,${ }^{80}$ binding modes ${ }^{81}$ or ion sizes. ${ }^{81}$ Representative examples are shown in Fig. 11. It is worth pointing out that sensor L2 showed an extremely low detection limit for $\mathrm{Cd}^{2+}(0.24 \mathrm{nM})$, and that both $\mathbf{L 1}$ and $\mathbf{L} 2$ were tested on solid supports, as the active components of indicator paper. ${ }^{82}$ In the case of HQZn, the cation-dependent emission colour of this TPA sensor, together with the higher affinity of the sensor for $\mathrm{Cd}^{2+}$ compared to $\mathrm{Zn}^{2+}$, permitted the authors to use the $\mathrm{Zn}$-chelated ligand as a $\mathrm{Cd}^{2+}$ sensor, eliminating the recurrent problem of $\mathrm{Zn}^{2+}$ interference during $\mathrm{Cd}^{2+}$ detection in biological systems. ${ }^{83}$

3.1.2. Reactivity-based ICT bicolour sensors: chemodosimeters. Highly efficient ratiometric probes for cations based on ICT can also be constructed relying on cationinduced irreversible bond-cleavage/bond-formation reactions on the molecular probe (Fig. 2, pattern B). Such cationmediated chemical changes in the probe can produce an enhancement or depletion of the charge-transfer process, which eventually affects the colour of the fluorescence emission. The examples range from probes based on the chemical reactivity of the cation with specific functional groups to form a cationcoordinated ICT fluorophore, ${ }^{84,85}$ to those based on cationmediated umpolung reactions on the probe (usually protection/ deprotection of functional groups) releasing a cation-free ICT fluorophore. ${ }^{86-92}$ Selected examples are shown in Fig. 12. One of the earliest examples was developed by Lin et al. in $2009 .^{88}$ The probe was based on a phenanthroimidazole dye containing an appended electron-rich 1,3-dithiane. $\mathrm{Cu}^{2+}$-Promoted desulfurization of the thioacetal rendered a formyl EWG, with the 

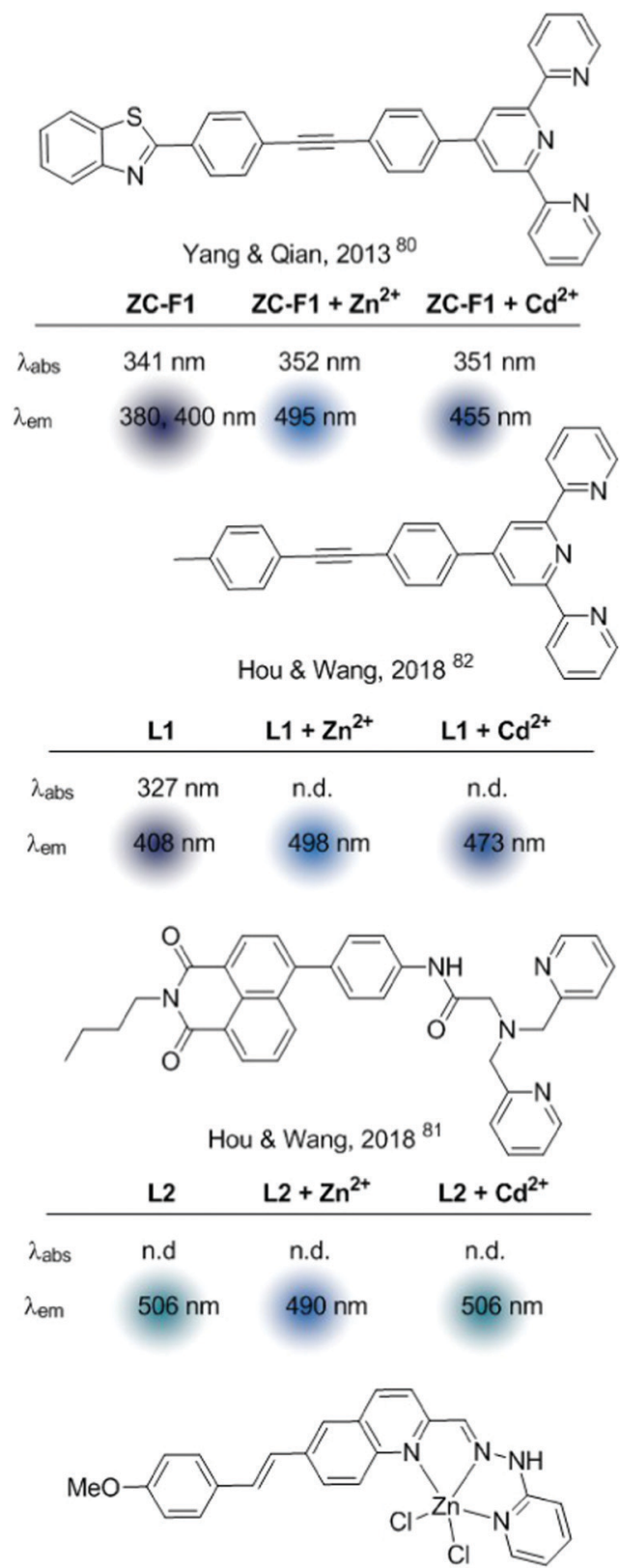

Meng \& Zhu, $2014^{83}$

\begin{tabular}{cccc} 
& \multicolumn{1}{c}{ HQZn } & HQZn $+\mathbf{Z n}^{2+}$ & HQZn $+\mathrm{Cd}^{2+}$ \\
\cline { 2 - 4 } abs & $355 \mathrm{~nm}$ & $394 \mathrm{~nm}$ & $430 \mathrm{~nm}$ \\
$\lambda_{\mathrm{em}}$ & $460 \mathrm{~nm}$ & $485 \mathrm{~nm}$ & $560 \mathrm{~nm}$ \\
$\delta_{2 \mathrm{PA}}(730 \mathrm{~nm})$ & n.d. & $48 \mathrm{GM}$ & $85 \mathrm{GM}$
\end{tabular}

Fig. 11 Multi-target sensors based on ICT.

concomitant bathochromic shift in the fluorescence emission due to ICT between the phenanthroimidazole (ERG) and the formed aldehyde (EWG). More recently, an acyclic thioacetal version of the same probe has been published for $\mathrm{Hg}^{2+}$-sensing. ${ }^{87}$ This cationmediated deprotection strategy has been extended to the construction of a large number of ICT-based chemodosimeters using different fluorescent dyes (dansyl, coumarin or naphthalimide, among others) (see examples in Fig. 12). ${ }^{86,88-92}$ Among these probes, one can distinguish two types depending on the effect (ICT enhancement or depletion) that the cation-induced reactivity produces on the probe. The former (metal-induced ICT enhancement) are a priori more appropriate for TPA applications. In a very recent example, Kim and Yoon developed NAP-PS (Fig. 12), a TPA probe for sensing intracellular $\mathrm{Hg}^{2+}$ in live cells and tissues. ${ }^{86}$ In this example, the presence of $\mathrm{Hg}^{2+}$ triggers the cleavage of the thiophosphinate $\mathrm{P}-\mathrm{O}$ bond, forming the ICT fluorophore NAP-O ${ }^{-}$. Additionally, grinding solid NAP-PS in the presence of $\mathrm{Hg}\left(\mathrm{ClO}_{4}\right)_{2}$ also produced a red-shift in the fluorescence emission of the resulting powder, demonstrating its response even in the solid state (Fig. 13).

All chemodosimeters described above are based on metalinduced bond-cleavage reactions. Alternatively, they can also be constructed using the so-called "covalent assembly principle". It refers to those probes in which the analyte triggers a cascade reaction on the probe, thus furnishing the conjugated backbone of a push-pull fluorescent dye. ${ }^{93}$ A coumarin-based probe (ATC-Hg), shown in Fig. 14, is a recent example of such a strategy. ${ }^{94} \mathrm{Hg}^{2+}$ induced the deprotection of 1,3-dithiolane to a formyl group that condensates intramolecularly with the nearby amino group. This cascade reaction renders a heterocyclic aromatic donor- $\pi$-acceptor ICT fluorescent dye. This probe showed high selectivity to $\mathrm{Hg}^{2+}$ and $\mathrm{MeHg}^{+}$, with sensitivities of $27 \mathrm{nM}$ and $5.8 \mu \mathrm{M}$, respectively.

Ratiometric sensors can also be constructed using metalinduced cascade reactions that reduce the conjugation on the probe. The group of Emrullahoğlu employed this strategy for the construction of BODIPY-based ratiometric probes for sensing $\mathrm{Au}^{3+}$ and $\mathrm{Hg}^{2+}$ (Fig. 15). ${ }^{95,96}$ Although strictly speaking these sensors cannot be considered ICT-based probes, it is convenient to include them here under the chemical pattern $\mathrm{B}$ shown in Fig. 2. In these examples, metal-mediated activation of the triple bond and subsequent intramolecular cyclisation and protonolysis generates the corresponding unconjugated BODIPY-furan fluorophores, as evidenced by the characteristic green emission after the cation-promoted reaction.

\subsection{Excimer/exciplex-based sensors}

An excimer was defined by Birks as "a dimer which is associated in an excited electronic state and which is dissociative (i.e. would dissociate in the absence of external restraints) in its ground electronic state" 97 (Fig. 4). Therefore, excimer formation is an irradiation-induced phenomenon, strongly dependent on the fluorophore-fluorophore distance. In general, in this kind of bicolour sensor the fluorophore is a polyaromatic system, being pyrene the one most frequently used. Since excimer emission is often red-shifted compared to the fluorescence of the monomer, excimer-based sensors can be used for the construction of ratiometric probes. This type of molecular sensor is designed in such a manner that the interaction of the analyte with the recognition unit of the probe alters the fluorophore-fluorophore distance. Based on this premise, one can distinguish between two types of probes: (A) intermolecular excimers formed by cation-assembled dimers, and (B) intramolecular excimers based 

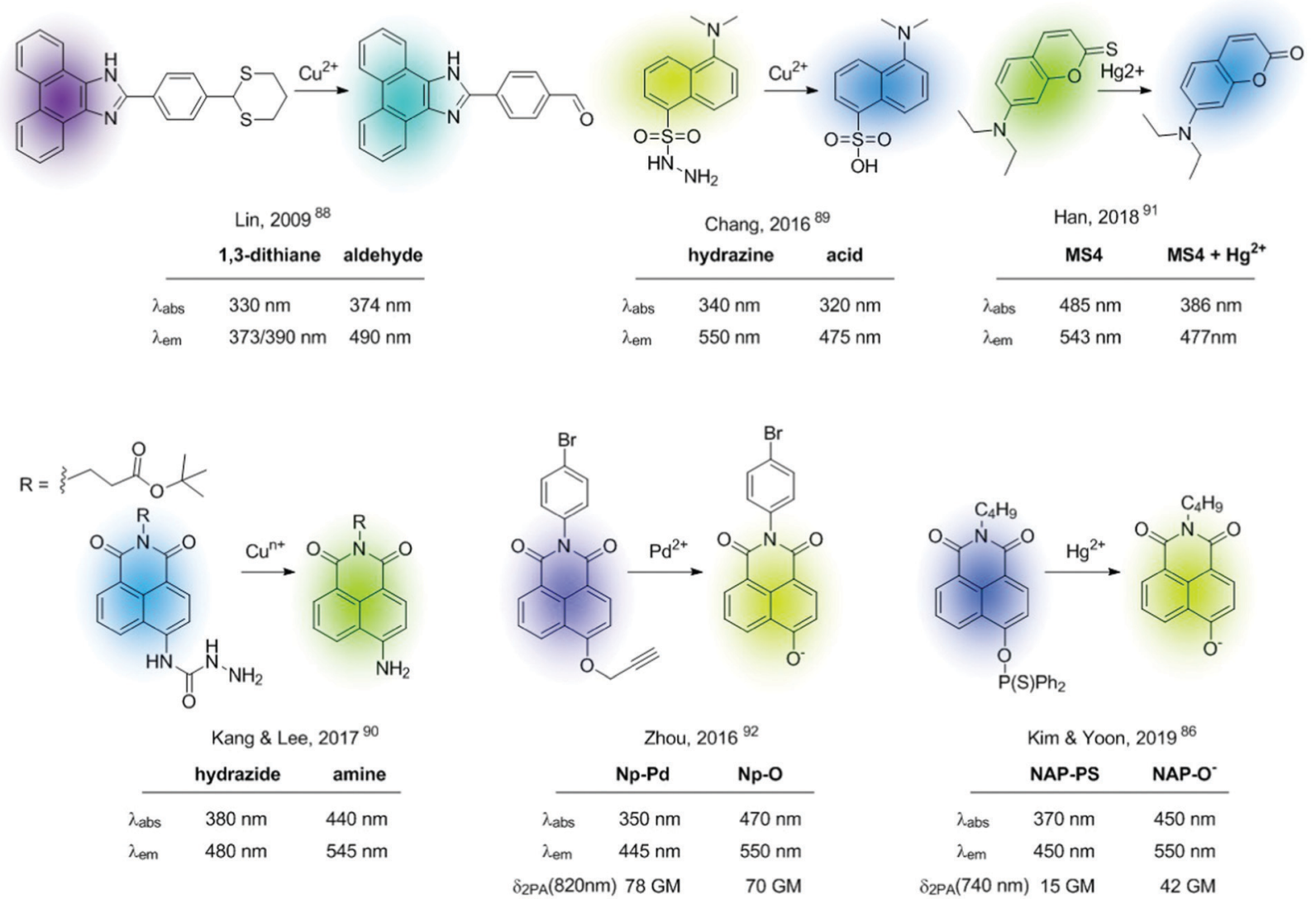

Fig. 12 Representative examples of fluorescent chemodosimeters for cations.

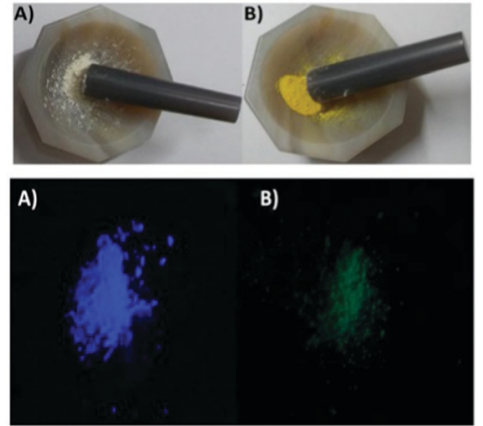

Fig. 13 NAP-PS chemodosimeter before (A) and after grinding with $\mathrm{Hg}\left(\mathrm{ClO}_{4}\right)_{2}$ (B). Reproduced from ref. 86 with permission from The Royal Society of Chemistry, copyright 2019.

on probes containing two fluorophores whose conformation is altered by cation binding. ${ }^{98}$

Some recent examples of sensors of type A are shown in Fig. 16. ${ }^{99-102}$ Within this configuration, the binding of two sensors to the cation places the fluorophores close enough to form an intramolecular excimer. Among the chosen examples, it is worth highlighting the molecular sensor developed by Misra et al. in $2015,{ }^{99}$ which was modified and immobilized onto silica supports showing a ratiometric response to $\mathrm{Hg}^{2+}$ in the solid-state. Additionally, the interaction can be reversed by

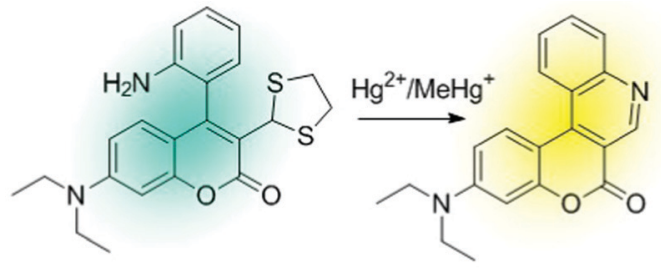

Li \& Yu, $2018^{94}$

\begin{tabular}{lll} 
& ATC-Hg & ATC-Hg $+\mathbf{H g}^{\text {n+ }}$ \\
\hline$\lambda_{\text {abs }}$ & $405 \mathrm{~nm}$ & $480 \mathrm{~nm}$ \\
$\lambda_{\text {em }}$ & $492 \mathrm{~nm}$ & $572 \mathrm{~nm}$
\end{tabular}

Fig. 14 Chemodosimeter based on covalent assembly.

treatment of the silica-supported sensor with EDTA allowing their use as $\mathrm{Hg}^{2+}$ extractors.

An early example of intramolecular excimer-based sensors of type $\mathrm{B}$ is the $\mathrm{Ba}^{2+}$ and $\mathrm{Sr}^{2+}$ probe developed by Ushakov and Barigelletti in 1999 (Fig. 17). ${ }^{103}$ The probe consists of a bis(styryl) fluorophore containing two crown ether units. It relies on the formation of intramolecular sandwich complexes with the sensing ions (with radius larger than the cavity size of the crown ether), which facilitates excimer emission from the close-lying fluorophores (Fig. 17). Although in the example the fluorescence of the $\mathrm{Ba}^{2+}$-complexed probe presents a blue-shifted 


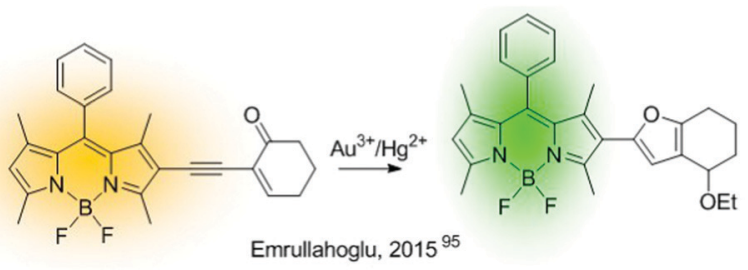

$$
\begin{array}{ccc} 
& \text { BURAK-1 } & \text { BOD-FUR } \\
\hline \lambda_{\text {abs }} & 526 \mathrm{~nm} & 504 \mathrm{~nm} \\
\lambda_{\text {em }} & 562 \mathrm{~nm} & 516 \mathrm{~nm}
\end{array}
$$

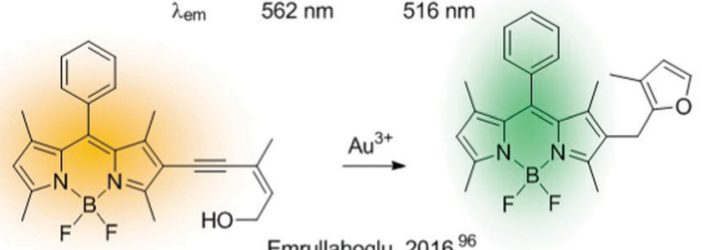

Emrullahoglu, $2016^{96}$

\begin{tabular}{ccc} 
& BOD-Z-EN & BOD-FUR' $^{\prime}$ \\
\hline$\lambda_{\text {abs }}$ & $532 \mathrm{~nm}$ & $503 \mathrm{~nm}$ \\
$\lambda_{\mathrm{em}}$ & $575 \mathrm{~nm}$ & $512 \mathrm{~nm}$
\end{tabular}

Fig. 15 Example of ratiometric probes based on metal-induced cascade reactions

band compared to that of the unbound probe, a strong fluorescence attributed to excimer formation was also observed at $c a$. $600 \mathrm{~nm}$.

In 2017 Lee et al. developed a ratiometric sensor for $\mathrm{Zn}^{2+}$ based on an intramolecular excimer inhibition. ${ }^{104}$ It consists of a symmetric pyrene-peptidyl probe. In this example, the

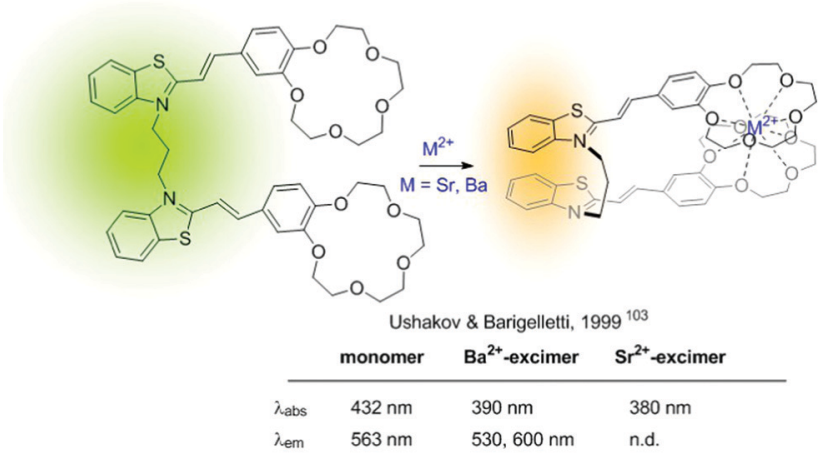

Fig. 17 Intramolecular excimer-based probe for $\mathrm{Ba}^{2+}$.

interaction of the probe with the cation modifies its conformation inhibiting pyrene-pyrene excimer formation (Fig. 18).

\subsection{FRET-based sensors}

Since the German scientist T. Förster described the FRET effect in $1948,{ }^{105}$ many fluorescent sensors based on this effect have been developed, ${ }^{42,106}$ which have been widely applied in diverse fields, such as biology, physiology, medicine, and pharmacology. This type of sensor presents ideal radiometric sensing properties as it benefits from a simple structural design. This section describes some FRET-based fluorescent sensors and the combination of these with other mechanisms. As described in Fig. 5, these sensors are based on an energy donor/acceptor system. In many of them, rhodamine appears as a fundamental part of the fluorescent sensor
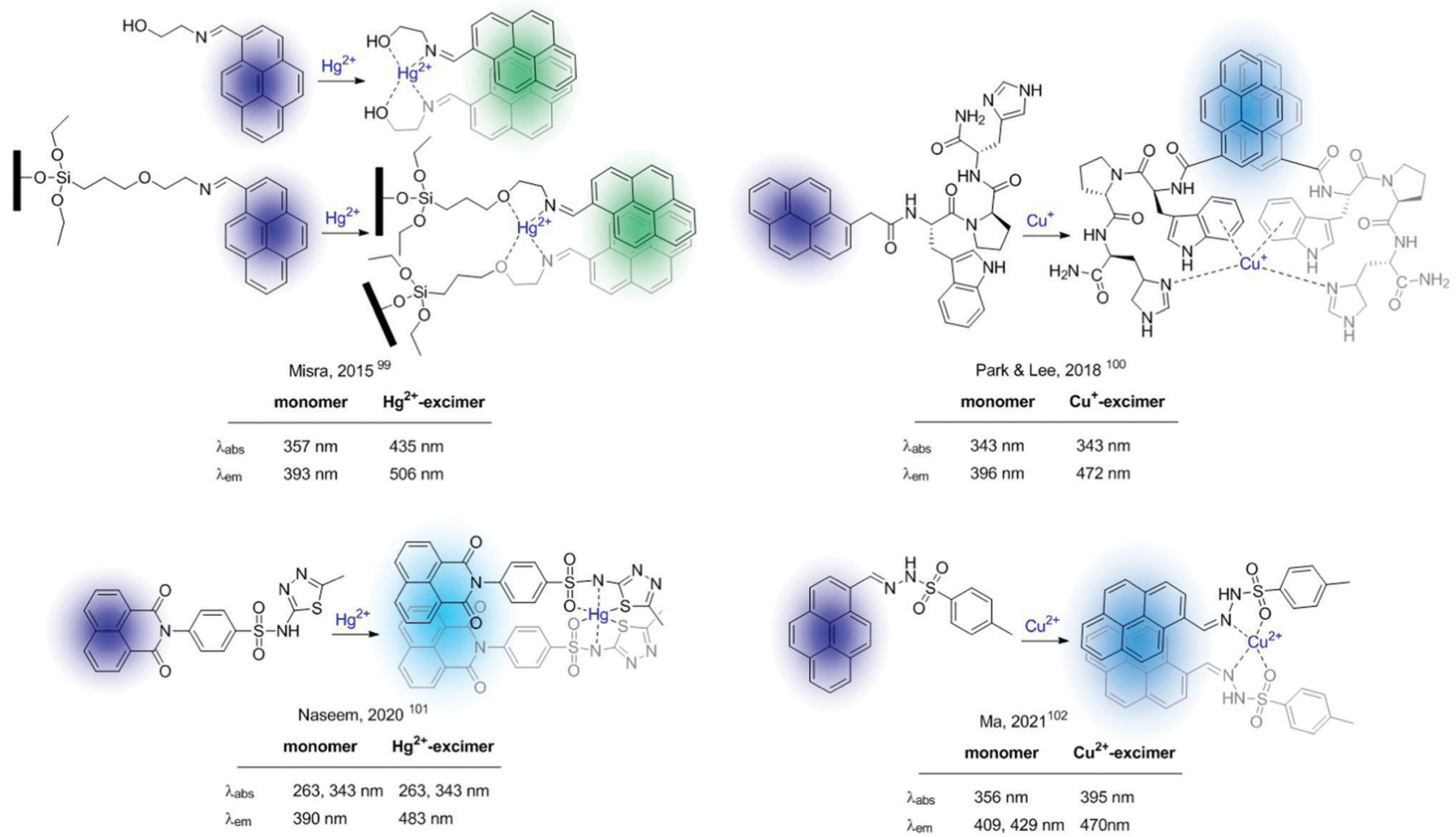

Fig. 16 Excimer-based probes for cations based on 2:1 sensor/cation supramolecular complexes. 


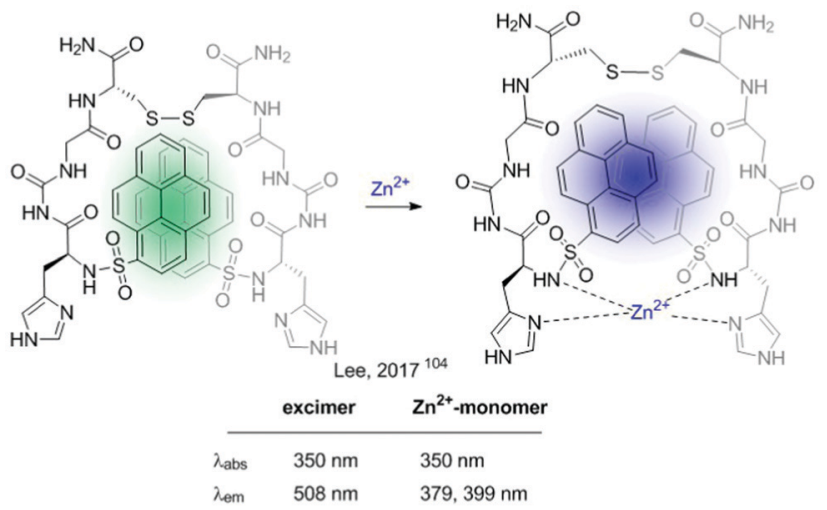

Fig. 18 Intramolecular excimer inhibition-based probe for $\mathrm{Zn}^{2+}$.

(acceptor), and it is integrated in the sensor in the form of the nonfluorescent rhodamine spirolactam. The analyte's reaction promotes spiro opening, revealing rhodamine fluorescent dye, which results in a FRET-induced rhodamine-centered emission.

In this context, Meng described in 2018 a two-photon radiometric fluorescent probe (RN3) for $\mathrm{Pd}^{2+}$ based on coumarin (donor) and rhodamine B (acceptor) connected by a diethylenetriamine linker (Fig. 19). ${ }^{107}$ The emission of a 7-substituted coumarin was determined in the range of $370-650 \mathrm{~nm}$, which fully covered the rhodamine B's absorption (450-590 nm). RN3 showed high sensitivity and selectivity towards $\mathrm{Pd}^{2+}$ in aqueous solutions, with a clear ratiometric fluorescent response in a

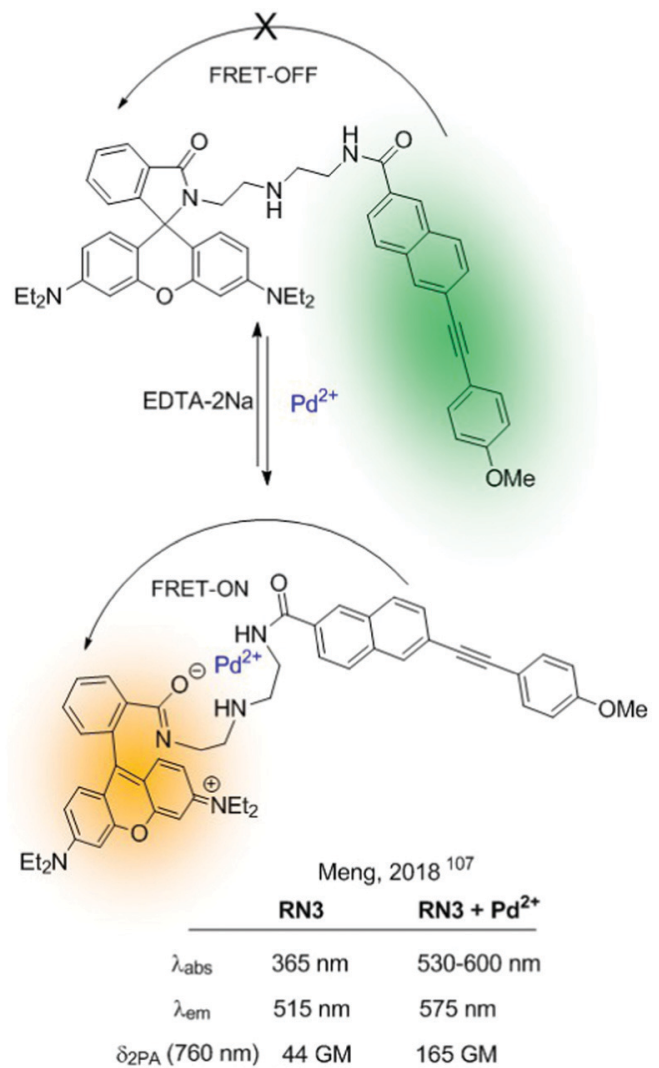

Fig. 19 RN3 based on the FRET-effect to detect $\mathrm{Pd}^{2+}$ in aqueous media.

one- and two-photon excitation regime. Additionally, interactions with the cation boosted the TPA cross-section, which was attributed to ICT enhancement on the coumarin upon coordination to $\mathrm{Pd}^{2+}$. $\mathbf{R N} 3$ was tested for the detection of $\mathrm{Pd}^{2+}$ in deep tissues and living cells under TPA, showing low cytotoxicity.

Different rhodamine-based FRET-dyads have been used for the construction of ratiometric sensors (i.e. using BODIPY or quinoline-benzothiazole as a donor). They have been widely used for sensing highly polluting cations such as $\mathrm{Hg}^{2+108}$ or $\mathrm{Cd}^{2+}$. For the latter, in 2015 Goswami described RQBT, a rhodamine/quinoline-benzothiazole-based sensor (Fig. 20). ${ }^{109}$ The interaction of $\mathrm{Cd}^{2+}$ with the probe results in the cleavage of the spiro ring and formation of the $\mathrm{Cd}^{2+}$-chelated structure, as confirmed by single-crystal X-ray analysis. Probe RQTB also showed ratiometric fluorescence in dry media (silica-gel TLC plates), which was used for the construction of portable $\mathrm{Cd}^{2+}$ sensing kits (Fig. 21).

Alternatively, FRET-probes for cations can be constructed by introducing an ICT sensor as part of a FRET dyad. Interaction of the cation with the ICT sensor will produce a shift in its absorption and/or emission properties, modifying the efficiency

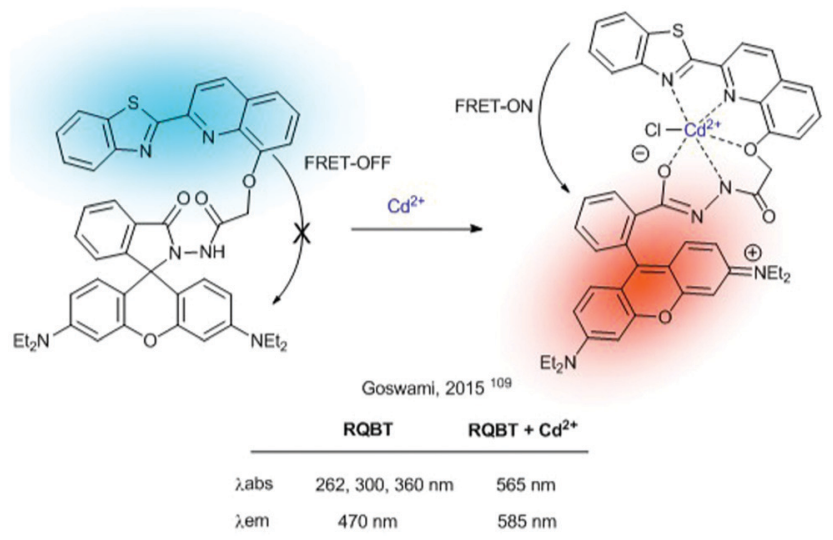

Fig. 20 Proposed FRET-based $\mathrm{Cd}^{2+}$-sensing mechanism of RQBT.

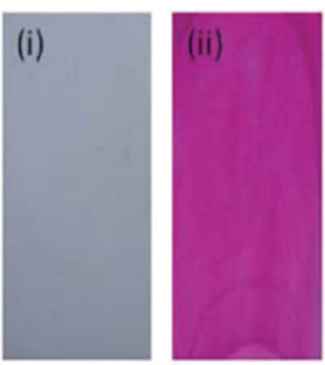

(a)

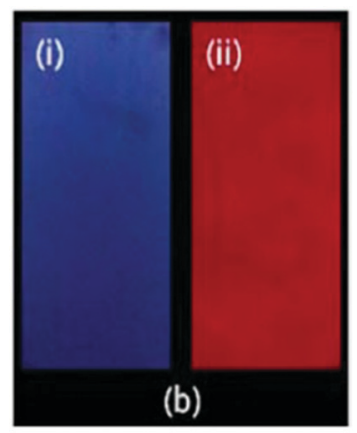

Fig. 21 Photographs of TLC plates after immersion in an RQBT-MeOH solution (i) and after immersion in an RQBT- $\mathrm{Cd}^{2+}$ methanol solution (ii) taken in ambient light (a) and under hand-held UV light (b). Excitation wavelength of the UV light is $365 \mathrm{~nm}$. Adapted from ref. 109 with permission from American Chemical Society, copyright 2015. 
of the FRET process. A recent example of such a strategy is the CPBT probe for $\mathrm{Zn}^{2+}$ developed by Chen, He, Guo and colleagues. ${ }^{110}$ CPBT integrates a TPA donor coumarin derivative with an ICT fluorophore 4-amine-7-sulfamoylbenzo[c][1,2,5]oxadiazole linked to the ionophore (Fig. 22). Within this type of construction, the interaction of the cation with the ICT fluorophore will cause a blue-shift in its emission, diminishing the spectral overlap between the donor and acceptor and weakening the FRET process. CPBT was tested for 2D and 3D ratiometric mapping of $\mathrm{Zn}^{2+}$ in living cells and in the head of zebrafish. Additionally, it showed TPA cross-section >40 GM, which makes it a suitable candidate to be used in two-photon excited fluorescence imaging.

CPBT can be described as an FRET-on $\rightarrow$ FRET-off ratiometric probe. The reverse mechanism (FRET-off $\rightarrow$ FRET-on) can also be envisioned, and the QA sensor, developed by Mao et al., serves as a representative example (Fig. 23). ${ }^{111}$ This probe contains an anthracene unit as a donor and a quinoline ICT sensor as an acceptor. In the absence of the analyte $\left(\mathrm{Zn}^{2+}\right)$, the probe shows the characteristic emission of anthracene. Interactions of $\mathrm{Zn}^{2+}$ with the quinolone sensor produce a red shift in its absorption band, together with a concomitant enhancement of the effective overlap with the anthracene emission, thus triggering the FRET process.

Closely related to FRET sensors, one can encounter the TBET-based probes (through bond energy transfer). ${ }^{112}$ They comprise sensors based on donor-acceptor dyads in which the donor and acceptor are connected through a conjugated bond. These probes can be used for the construction of sensors
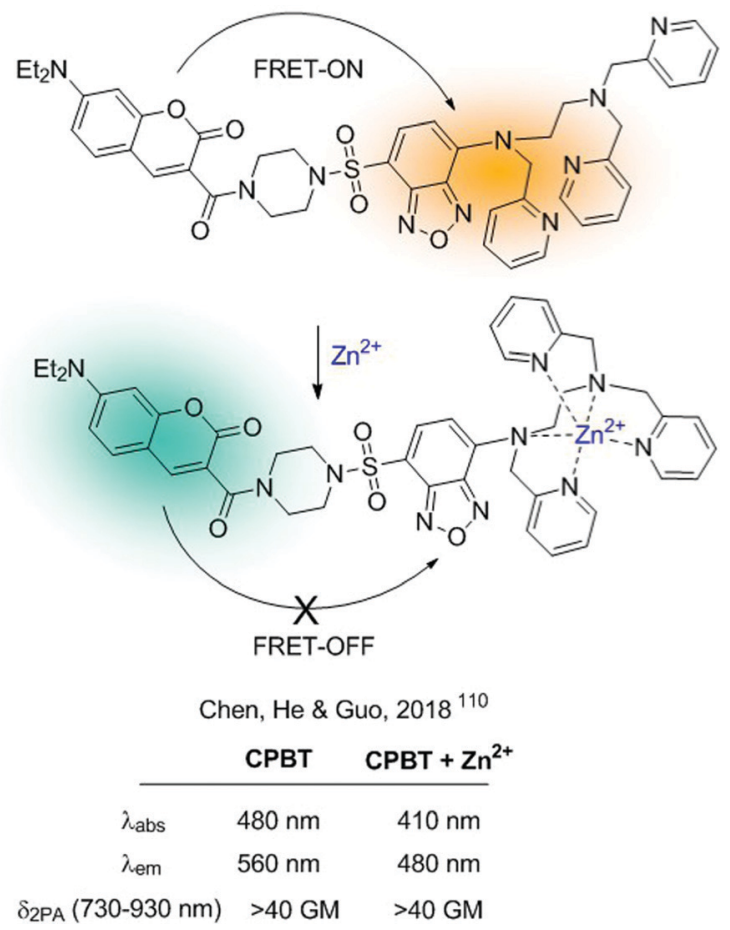

Fig. 22 FRET-ICT probe for $\mathrm{Zn}^{2+}$, showing an FRET-ON/FRET-OFF mechanism.

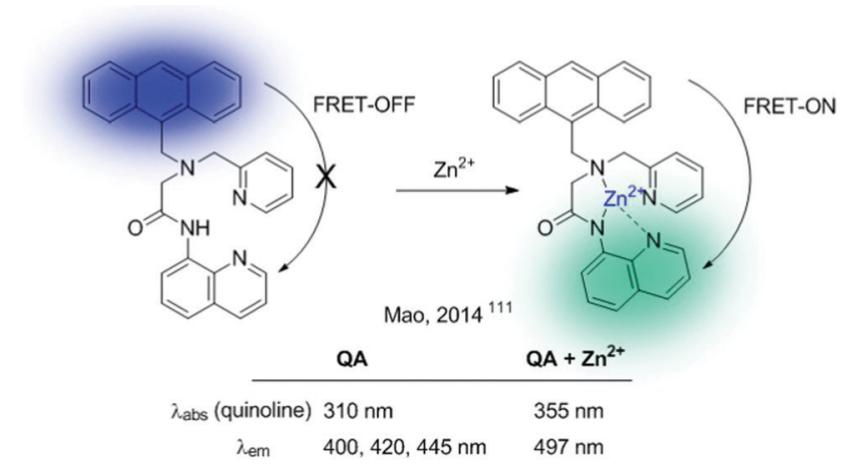

Fig. 23 FRET-ICT probe for $\mathrm{Zn}^{2+}$, showing an FRET-OFF/FRET-ON mechanism.

as long as the cation interaction alters the extent of the FRET process. The simplest strategy consists of revealing a "dormant" fluorophore (rhodamine), as described before for FRET probes. A representative example is $\mathbf{N p - R h}$, a TPA ratiometric TBETbased probe for $\mathrm{Cu}^{2+}$ shown in Fig. $24 .^{113}$

\subsection{Two-component fluorescent bicolour indicators}

We applied the concept outlined in Section 2. 4 to the issue associated with the background-free detection of individual barium cations in $\beta \beta 0 v$ experiments (vide supra). Application of indicators to this kind of problem requires a series of developments to enable its use. Although several molecular fluorescent sensors for $\mathrm{Ba}^{2+}$ capture and detection have been described in solution, ${ }^{114-120}$ the features of the $\mathrm{Xe} \rightarrow \mathrm{Ba}^{2+}+2 \mathrm{e}$ experiment at a high pressure require the development of fluorescent sensors able to operate efficiently in the interface between a solid substrate and ultra-dry noble gas.

The Xenon Chamber must incorporate "dry" molecular sensors capable of capturing the $\mathrm{Ba}^{2+}$ ion in a pressurised xenon environment. In addition, a very stable binding that prevents release of the barium cation before optical interrogation is required. As we have commented above, one possibility consists of developing monocolour ICT turn-on sensors. ${ }^{23,24}$ Our approach $^{121}$ was based on the above-mentioned two-component design. Benzo $[a]$ imidazo[5,1,2-cd]indolizine was chosen as a suitable fluorophore of type $\mathbf{C} 3$ (Fig. 1). To this HetAr ${ }_{1}$ group a para-phenylene group was added as an $\mathrm{Ar}_{2}$ component. Finally, a pentaoxa-16-azacyclooctadecane aza-crown ether unit was included as a $\mathbf{B} 3$ type coronand ionophore. This compound was named FBI, Fluorescent Bicolour Indicator (Fig. 25A). DFT calculations showed that the HetAr $\mathrm{r}_{1}-\mathrm{Ar}_{2}$ group gives rise to the fluorophore 1 ensemble, in which the dihedral angle formed by both components was close to coplanarity.

Capture of $\mathrm{Ba}^{2+}$ by adding barium perchlorate in acetonitrile solution or in the gas phase led to a complex in which the $\mathrm{Ba}^{2+} \ldots \mathrm{Ph}$ interaction disconnects both components of fluorophore 1 (Fig. 25B). This coordination pattern in which the barium cation, aside the cation $-\pi$ interaction, complexes the five oxygen atoms of the aza-crown ether and one nitrogen atom of fluorophore 2 was elucidated by DFT calculations and NMR experiments. ${ }^{121}$ As expected, the $\mathbf{F B I} \cdot \mathbf{B a}^{2+}$ complex shows a 

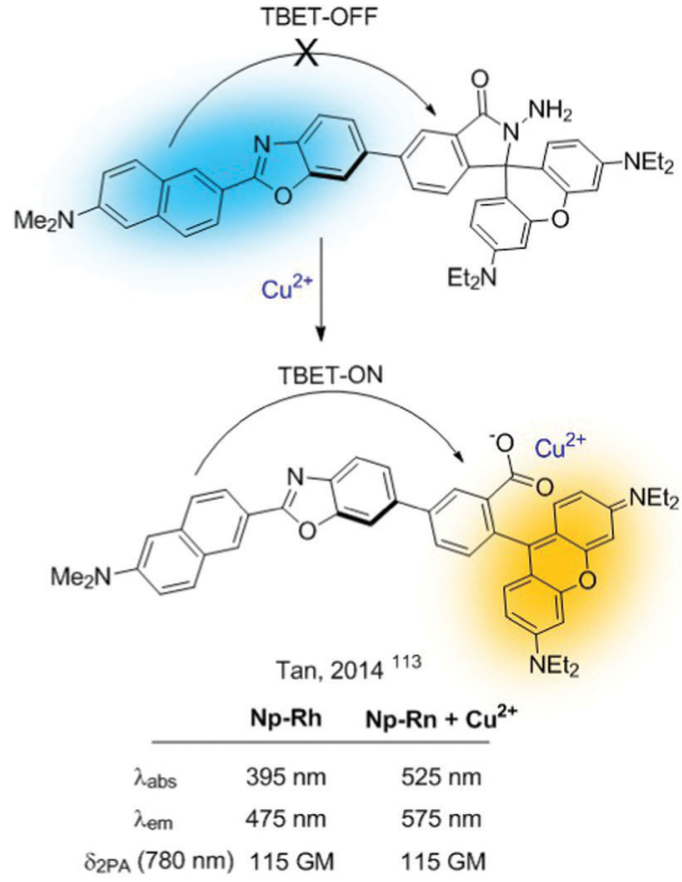

Fig. 24 TBET-based probe for sensing $\mathrm{Cu}^{2+}$

noticeable blue shift (Fig. 25C), with a peak discrimination factor $f_{\lambda}=179.74$ and a brightness value of $B_{\lambda}=8.06 \mathrm{M}^{-1} \mathrm{~cm}^{-1}$. The fluorescence spectra in solution and in the gas phase show intense emission signals for FBI and $\mathbf{F B I} \cdot \mathbf{B a}^{\mathbf{2}^{2+}}$ (Fig. 25D) compatible with the FMO scheme shown in Fig. 25. Application of an optical filter for $\lambda>450 \mathrm{~nm}$ to both compounds shows a no signal pattern in the case of FBI. This separation of the chelated/unchelated photophysical properties results fundamental for $\beta \beta 0 \nu$ experiments. In order to detect the $\mathrm{Ba}^{2+}$ cations produced by the decay, they will be driven by electric fields to the cathode where a region of a few centimetres will need to be scanned to look for a positive signal of the complexed molecule. This region will be fully covered, with a density of $10^{5}-10^{6}$ molecules $\mu \mathrm{m}^{-2}$, by the bicolour indicator to guarantee a high efficiency to capture the cation. The discrimination factor between the response of the complexed indicator and the response of the residual background molecules on the surface must be large to allow separation of the single chelated molecule from the background emission produced by the surrounding molecules that will also be illuminated during the scan.

\section{Conclusions and outlook}

In this paper, we have highlighted significant advances in the design and experimental validation of fluorescent bicolour sensors. Most of these photo-indicators are based on binding ionophores and fluorophores connected by covalent bonds. If required, suitable spacer units and linkers are incorporated. In addition, some fluorescent bicolour sensors merge the ionophore and the fluorophore components, thus yielding chelating fluorophores whose photophysical properties change
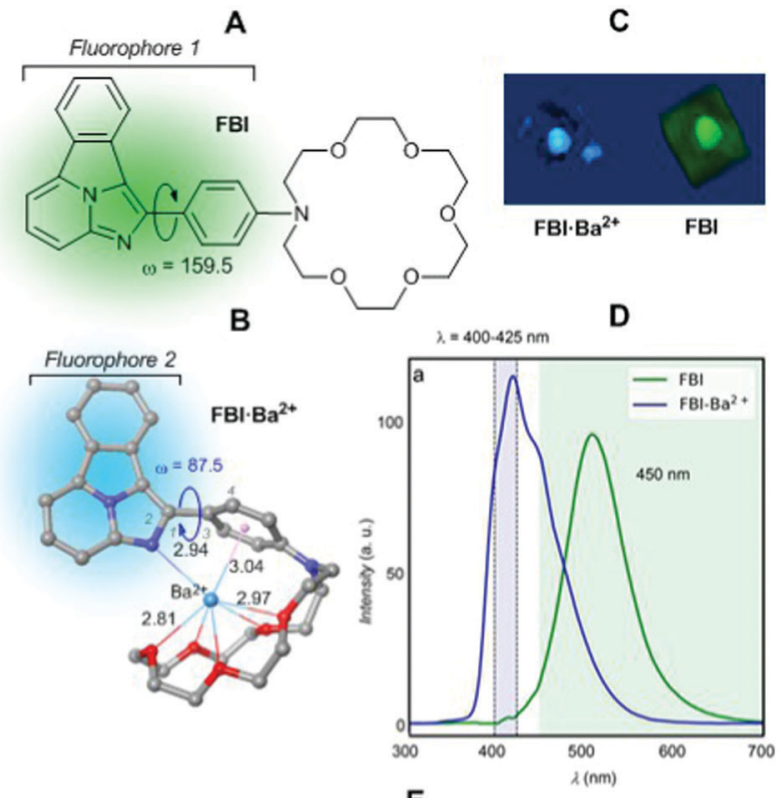

E
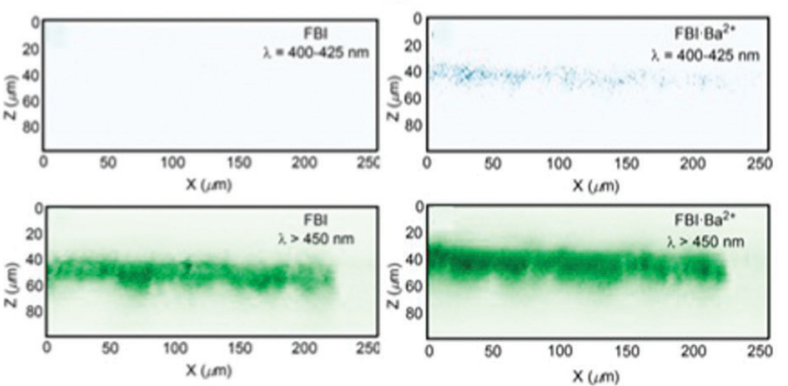

Fig. 25 (A) Structure of the FBI molecule showing the approximate coplanarity of the two components of fluorophore 1. (B) DFT optimized structure of complex $\mathbf{F B I} \cdot \mathbf{B a}^{2+}$. Bond angles and distances are given in deg and $\AA$, respectively. (C) $3 \mathrm{D}$ tomography of $\mathrm{FBI}$ and $\mathrm{FBI} \cdot \mathrm{Ba}^{2+}$ obtained by means of a two-photon absorption (TPA) microscopy device. (D) Solidgas phase emission spectra of $\mathbf{F B I}$ and $\mathbf{F B I} \cdot \mathbf{B a}^{2+}$ adsorbed on silica. (E) Z-X profiles showing emission profiles (after TPA excitation) from both complexes with and without a $\lambda>450 \mathrm{~nm}$ filter. Adapted from ref. 121 with permission from Springer Nature, copyright 2020.

significantly upon cation binding. In other cases, the ionophore (most frequently a coronand) and the fluorophore work together upon cation coordination and induce a bathochromic or hypsochromic shift in their emission spectra. Finally, several fluorescent sensors generate their bicolour response by means of a chemical reaction promoted by the metallic cation that induces a change in the chemical structure of the fluorophore.

These structural and chemical features result in a bicolour response as an attractive alternative to on-off or off-on changes via photoinduced electron transfer (PET) phenomena upon cation coordination. Instead, the bicolour response requires changes in the photophysical properties of the sensor, which is fluorescent in both the free and coordinated states within an on-on' conceptual scheme. These dual patterns include intramolecular charge transfer processes (ICT), excimer/exciplex inter- or intramolecular complexes and Förster resonance energy transfer (FRET). As an additional design feature, the 
fluorophore can incorporate different rotatable units that can be decoupled in the presence of a suitable metal cation thus resulting in different fluorophores in the free and coordinated states.

It is expected that in the years to come these designs will permit the development of more sensitive and selective fluorescent bicolour sensors able to detect specifically metal cations in very low concentrations. This situation will permit further advances in fields ranging from cell biology to particle physics. In addition, the development of fluorescent sensors able to detect metallic cations in the gas phase and at solid-gas interfaces would permit the exploration of supramolecular chemistry in the absence of solvents, thus expanding the field from "wet" to "dry" chemistry.

\section{Conflicts of interest}

There are no conflicts to declare.

\section{Acknowledgements}

This work was supported by the Basque Government (Grants IT1346-19 and IT1180-19), by the Spanish Ministry of Science and Innovation (MICINN-FEDER, Grants PID2019-104772GB-I00, PID2019-111281GB-I00, RED2018-102387-T, and RED2018102471-T), and by the European Research Council (ERC) under the European's Union Horizon 2020 research and innovation programme (Grant agreement ERC-2020-SyG 951281).

\section{References}

1 A. P. de Silva, T. S. Moody and G. D. Wright, Analyst, 2009, 134, 2385-2393.

2 L. Basabe-Desmonts, D. N. Reinhoudt and M. CregoCalama, Chem. Soc. Rev., 2007, 36, 993-1017.

3 N. De Acha, C. Elosua, J. M. Corres and F. J. Arregui, Sensors, 2019, 19, 599, DOI: 10.3390/s19030599.

4 K. P. Carter, A. M. Young and A. E. Palmer, Chem. Rev., 2014, 114, 4564-4601.

5 J. J. Gómez-Cadenas, J. Martin-Albo, M. Mezzetto, F. Monrabal and M. Sorel, Riv. Nuovo Cimento Soc. Ital. Fis., 2012, 35, 29-98.

6 W.-W. Zhao, J.-J. Xu and H.-Y. Chen, Analyst, 2016, 141, 4262-4271.

7 L. Eddaif, A. Shaban and J. Telegdi, Int. J. Environ. Anal. Chem., 2019, 99, 824-853.

8 B. Valeur and M. N. Berberan-Santos, in Molecular Fluorescence, ed. B. Valeur and M. N. Berberan-Santos, 2012, pp. 409-478.

9 B. Valeur and I. Leray, Coord. Chem. Rev., 2000, 205, 3-40. 10 L. Yu, Y. Qiao, L. Miao, Y. He and Y. Zhou, Chin. Chem. Lett., 2018, 29, 1545-1559.

11 A. P. de Silva, H. Q. N. Gunaratne, T. Gunnlaugsson, A. J. M. Huxley, C. P. McCoy, J. T. Rademacher and T. E. Rice, Chem. Rev., 1997, 97, 1515-1566.
12 S. Chowdhury, B. Rooj, A. Dutta and U. Mandal, J. Fluoresc., 2018, 28, 999-1021.

13 N. S. Patil, R. B. Dhake, M. I. Ahamed and U. Fegade, J. Fluoresc., 2020, 30, 1295-1330.

14 L. Zhu, L. Zhang and A. H. Younes, Supramol. Chem., 2009, 21, 268-283.

15 J. Yin, Y. Hu and J. Yoon, Chem. Soc. Rev., 2015, 44, 4619-4644.

16 D. G. Smith, I. L. Topolnicki, V. E. Zwicker, K. A. Jolliffe and E. J. New, Analyst, 2017, 142, 3549-3563.

17 M. Ahmed, M. Faisal, A. Ihsan and M. M. Naseer, Analyst, 2019, 144, 2480-2497.

18 D. Yoo, Y. Park, B. Cheon and M.-H. Park, Nanoscale Res. Lett., 2019, 14, 272.

19 L. Wang, W. Li, L. Yin, Y. Liu, H. Guo, J. Lai, Y. Han, G. Li, M. Li, J. Zhang, R. Vajtai, P. M. Ajayan and M. Wu, Sci. Adv., 2020, 6, eabb6772.

20 D. S. a. R. B. I. Grabchev, Curr. Med. Chem., 2012, 19, 4976-4983.

21 A. K. Jana and S. Natarajan, ChemPlusChem, 2017, 82, 1153-1163.

22 S. S. Tan, S. J. Kim and E. T. Kool, J. Am. Chem. Soc., 2011, 133, 2664-2671.

23 N. C. Shaner, P. A. Steinbach and R. Y. Tsien, Nat. Methods, 2005, 2, 905-909.

24 B. Valeur and M. N. Berberan-Santos, Molecular Fluorescence-Principles and Applications, Wiley-VCH, Weinheim (Germany), 2nd edn, 2012.

25 J. A. Kemlo and T. M. Shepherd, Chem. Phys. Lett., 1977, 47, 158-162.

26 A. Bigdeli, F. Ghasemi, S. Abbasi-Moayed, M. Shahrajabian, N. Fahimi-Kashani, S. Jafarinejad, M. A. Farahmand Nejad and M. R. Hormozi-Nezhad, Anal. Chim. Acta, 2019, 1079, 30-58.

27 R. Gui, H. Jin, X. Bu, Y. Fu, Z. Wang and Q. Liu, Coord. Chem. Rev., 2019, 383, 82-103.

28 J. Gierschner, S. K. Behera and S. Y. Park, Angew. Chem., Int. Ed., 2021, 60, DOI: 10.1002/anie.202009789.

29 D. R. Nygren, J. Phys.: Conf. Ser., 2015, 650, 012002.

30 E. Majorana, Il Nuovo Cimento (1924-1942), 2008, 14, 171.

31 A. D. Sakharov, Pis'ma Z. Eksp. Teor. Fiz., 1967, 5, 32-35.

32 M. Fukugita and T. Yanagida, Phys. Lett. B, 1986, 174, 45-47.

33 A. D. McDonald, B. J. P. Jones, D. R. Nygren, C. Adams, V. Álvarez, C. D. R. Azevedo, J. M. Benlloch-Rodríguez, F. I. G. M. Borges, A. Botas, S. Cárcel, J. V. Carrión, S. Cebrián, C. A. N. Conde, J. Díaz, M. Diesburg, J. Escada, R. Esteve, R. Felkai, L. M. P. Fernandes, P. Ferrario, A. L. Ferreira, E. D. C. Freitas, A. Goldschmidt, J. J. Gómez-Cadenas, D. González-Díaz, R. M. Gutiérrez, R. Guenette, K. Hafidi, J. Hauptman, C. A. O. Henriques, A. I. Hernandez, J. A. Hernando Morata, V. Herrero, S. Johnston, L. Labarga, A. Laing, P. Lebrun, I. Liubarsky, N. López-March, M. Losada, J. Martín-Albo, G. Martínez-Lema, A. Martínez, F. Monrabal, C. M. B. Monteiro, F. J. Mora, 
L. M. Moutinho, J. Muñoz Vidal, M. Musti, M. NebotGuinot, P. Novella, B. Palmeiro, A. Para, J. Pérez, M. Querol, J. Repond, J. Renner, S. Riordan, L. Ripoll, J. Rodríguez, L. Rogers, F. P. Santos, J. M. F. dos Santos, A. Simón, C. Sofka, M. Sorel, T. Stiegler, J. F. Toledo, J. Torrent, Z. Tsamalaidze, J. F. C. A. Veloso, R. Webb, J. T. White and N. Yahlali, Phys. Rev. Lett., 2018, 120, 132504.

34 P. Thapa, I. Arnquist, N. Byrnes, A. A. Denisenko, F. W. Foss, B. J. P. Jones, A. D. McDonald, D. R. Nygren and K. Woodruff, Sci. Rep., 2019, 9, 15097.

35 P. Thapa, N. K. Byrnes, A. A. Denisenko, J. X. Mao, A. D. McDonald, C. A. Newhouse, T. T. Vuong, K. Woodruff, K. Nam, D. R. Nygren, B. J. P. Jones and F. W. Foss, Jr., ACS Sens., 2021, 6, 192-202.

36 S.-H. Park, N. Kwon, J.-H. Lee, J. Yoon and I. Shin, Chem. Soc. Rev., 2020, 49, 143-179.

37 C. Guo, A. C. Sedgwick, T. Hirao and J. L. Sessler, Coord. Chem. Rev., 2021, 427, 213560.

38 M. H. Lee, J. S. Kim and J. L. Sessler, Chem. Soc. Rev., 2015, 44, 4185-4191.

39 X. Pei, Y. Pan, L. Zhang and Y. Lv, Appl. Spectrosc. Rev., 2020, 1-22, DOI: 10.1080/05704928.2020.1793770.

40 J. Fan, M. Hu, P. Zhan and X. Peng, Chem. Soc. Rev., 2013, 42, 29-43.

41 Y. Fu and N. S. Finney, RSC Adv., 2018, 8, 29051-29061.

42 R. Zhang, F. Yan, Y. Huang, D. Kong, Q. Ye, J. Xu and L. Chen, RSC Adv., 2016, 6, 50732-50760.

43 M. A. Haidekker and E. A. Theodorakis, J. Mater. Chem. C, 2016, 4, 2707-2718.

44 J. Li, D. Yim, W.-D. Jang and J. Yoon, Chem. Soc. Rev., 2017, 46, 2437-2458.

45 S. Fery-Forgues, M. T. Le Bris, J. P. Guette and B. Valeur, J. Phys. Chem., 1988, 92, 6233-6237.

46 H. G. Loehr and F. Voegtle, Acc. Chem. Res., 1985, 18, 65-72.

47 Z. Liu, S. K. M. Nalluri and J. F. Stoddart, Chem. Soc. Rev., 2017, 46, 2459-2478.

48 J.-F. Chen, Q. Lin, Y.-M. Zhang, H. Yao and T.-B. Wei, Chem. Commun., 2017, 53, 13296-13311.

49 M. Aginagalde, Y. Vara, A. Arrieta, R. Zangi, V. L. Cebolla, A. Delgado-Camón and F. P. Cossío, J. Org. Chem., 2010, 75, 2776-2784.

50 É. Lévesque, W. S. Bechara, L. Constantineau-Forget, G. Pelletier, N. M. Rachel, J. N. Pelletier and A. B. Charette, J. Org. Chem., 2017, 82, 5046-5067.

51 G. T. Hermanson, Bioconjugate Techniques, Academic Press Elsevier, London, UK, 2013.

52 J. Hoche, H.-C. Schmitt, A. Humeniuk, I. Fischer, R. Mitrić and M. I. S. Röhr, Phys. Chem. Chem. Phys., 2017, 19, 25002-25015.

53 A. Kaur, P. Kaur and S. Ahuja, Anal. Methods, 2020, 12, 5532-5550.

54 A. Kaur, P. Kaur and S. Ahuja, Anal. Methods, 2021, 13, 730.

55 J. R. Lakowicz, Principles of Fluorescence Spectroscopy, Springer, 3nd edn, 2006.
56 S. E. Braslavsky, Pure Appl. Chem., 2007, 79, 293-465.

57 B. Liu and G. C. Bazan, J. Am. Chem. Soc., 2006, 128, 1188-1196.

58 P. G. Wu and L. Brand, Anal. Biochem., 1994, 218, 1-13.

59 D. A. Dougherty, Acc. Chem. Res., 2013, 46, 885-893.

60 R. Misra and S. P. Bhattacharyya, Intramolecular Charge Transfer Theory and Applications, 2018.

61 G. Grynkiewicz, M. Poenie and R. Y. Tsien, J. Biol. Chem., 1985, 260, 3440-3450.

62 S. Grisendi, Nat. Cell Biol., 2009, 11, S15-S15.

63 H. Szmacinski, I. Gryczynski and J. R. Lakowicz, Photochem. Photobiol., 1993, 58, 341-345.

64 S. A. Garwin, M. S. Kelley, A. C. Sue, E. L. Que, G. C. Schatz, T. K. Woodruff and T. V. O'Halloran, J. Am. Chem. Soc., 2019, 141, 16696-16705.

65 D. Cheng, X. Liu, Y. Xie, H. Lv, Z. Wang, H. Yang, A. Han, X. Yang and L. Zang, Sensors, 2017, 17, 2517.

66 X. Jiao, C. Liu, S. He, L. Zhao and X. Zeng, Dyes Pigm., 2019, 160, 86-92.

67 V. Juvekar, S. J. Park, J. Yoon and H. M. Kim, Coord. Chem. Rev., 2021, 427, 213574.

68 D. Kim, H. G. Ryu and K. H. Ahn, Org. Biomol. Chem., 2014, 12, 4550-4566.

69 S. Sumalekshmy and C. J. Fahrni, Chem. Mater., 2011, 23, 483-500.

70 D. Bourassa, C. M. Elitt, A. M. McCallum, S. Sumalekshmy, R. L. McRae, M. T. Morgan, N. Siegel, J. W. Perry, P. A. Rosenberg and C. J. Fahrni, ACS Sens., 2018, 3, 458-467.

71 P. Ning, J. Jiang, L. Li, S. Wang, H. Yu, Y. Feng, M. Zhu, B. Zhang, H. Yin, Q. Guo and X. Meng, Biosens. Bioelectron., 2016, 77, 921-927.

72 W. Li, Z. Liu, B. Fang, M. Jin and Y. Tian, Biosens. Bioelectron., 2020, 148, 111666.

73 X. Meng, S. Wang, Y. Li, M. Zhu and Q. Guo, Chem. Commun., 2012, 48, 4196-4198.

74 K. P. Divya, S. Sreejith, P. Ashokkumar, K. Yuzhan, Q. Peng, S. K. Maji, Y. Tong, H. Yu, Y. Zhao, P. Ramamurthy and A. Ajayaghosh, Chem. Sci., 2014, 5, 3469-3474.

75 W. Li, B. Fang, M. Jin and Y. Tian, Anal. Chem., 2017, 89, 2553-2560.

76 L. Zhu, A. H. Younes, Z. Yuan and R. J. Clark, J. Photochem. Photobiol., A, 2015, 311, 1-15.

77 J. Bednarska, R. Zaleśny, N. Arul Murugan, W. Bartkowiak, H. Ågren and M. Odelius, J. Phys. Chem. B, 2016, 120, 9067-9075.

78 S. Huang, B.-Z. Yang and A.-M. Ren, J. Mol. Struct., 2016, 1114, 65-77.

79 D. Wang, A.-M. Ren, L.-Y. Zou, J.-F. Guo and S. Huang, J. Photochem. Photobiol., A, 2017, 341, 20-30.

80 Y. Tan, J. Gao, J. Yu, Z. Wang, Y. Cui, Y. Yang and G. Qian, Dalton Trans., 2013, 42, 11465-11470.

81 Y. Zhang, X. Chen, J. Liu, G. Gao, X. Zhang, S. Hou and H. Wang, New J. Chem., 2018, 42, 19245-19251.

82 Y.-Y. Zhang, X.-Z. Chen, X.-Y. Liu, M. Wang, J.-J. Liu, G. Gao, X.-Y. Zhang, R.-Z. Sun, S.-C. Hou and H.-M. Wang, Sens. Actuators, B, 2018, 273, 1077-1084. 
83 W. Ye, S. Wang, X. Meng, Y. Feng, H. Sheng, Z. Shao, M. Zhu and Q. Guo, Dyes Pigm., 2014, 101, 30-37.

84 J. Young Choi, G.-H. Kim, Z. Guo, H. Yeon Lee, K. M. K. Swamy, J. Pai, S. Shin, I. Shin and J. Yoon, Biosens. Bioelectron., 2013, 49, 438-441.

85 Z. Li, Y. Xu, J. Fu, H. Zhu and Y. Qian, Chem. Commun., 2018, 54, 888-891.

86 L. Chen, S. J. Park, D. Wu, H. M. Kim and J. Yoon, Chem. Commun., 2019, 55, 1766-1769.

87 J. Hu, Z. Hu, S. Liu, Q. Zhang, H.-W. Gao and K. Uvdal, Sens. Actuators, B, 2016, 230, 639-644.

88 W. Lin, L. Yuan, W. Tan, J. Feng and L. Long, Chem. - Eur. J., 2009, 15, 1030-1035.

89 M. G. Choi, J. Kim, J. M. Hong, I. J. Chang, S. Ahn and S.-K. Chang, Tetrahedron Lett., 2016, 57, 975-978.

90 S. Y. Park, W. Kim, S.-H. Park, J. Han, J. Lee, C. Kang and M. H. Lee, Chem. Commun., 2017, 53, 4457-4460.

91 S. Qin, B. Chen, J. Huang and Y. Han, New J. Chem., 2018, 42, 12766-12772.

92 L. Zhou, S. Hu, H. Wang, H. Sun and X. Zhang, Spectrochim. Acta, Part A, 2016, 166, 25-30.

93 Z. Zhang, B. Zhang, X. Qian, Z. Li, Z. Xu and Y. Yang, Anal. Chem., 2014, 86, 11919-11924.

94 S.-L. Pan, K. Li, L.-L. Li, M.-Y. Li, L. Shi, Y.-H. Liu and X.-Q. Yu, Chem. Commun., 2018, 54, 4955-4958.

95 M. Üçüncü, E. Karakuş and M. Emrullahoğlu, Chem. - Eur. J., 2015, 21, 13201-13205.

96 M. Üçüncü, E. Karakuş and M. Emrullahoğlu, Chem. Commun., 2016, 52, 8247-8250.

97 J. B. Birks, Rep. Prog. Phys., 1975, 38, 903-974.

98 Y. Hong, J. W. Y. Lam and B. Z. Tang, Chem. Soc. Rev., 2011, 40, 5361-5388.

99 S. S. Razi, R. Ali, P. Srivastava and A. Misra, RSC Adv., 2015, 5, 79538-79547.

100 P. K. Mehta, E.-T. Oh, H. J. Park and K.-H. Lee, Sens. Actuators, B, 2018, 256, 393-401.

101 B. Muzey and A. Naseem, J. Photochem. Photobiol., A, 2020, 391, 112354.

102 L.-J. Ma, Q. Liang, R. Feng, Z. Lv, F. Cui, L. Li, L. Yang, H. Liu and F. Sun, J. Photochem. Photobiol., A, 2021, 408, 113086.

103 E. N. Ushakov, S. P. Gromov, O. A. Fedorova, Y. V. Pershina, M. V. Alfimov, F. Barigelletti, L. Flamigni and V. Balzani, J. Phys. Chem. A, 1999, 103, 11188-11193.
104 P. K. Mehta, E.-T. Oh, H. J. Park and K.-H. Lee, Sens. Actuators, B, 2017, 245, 996-1003.

105 T. Förster, Ann. Phys., 1948, 437, 55-75.

106 L. Wu, C. Huang, B. P. Emery, A. C. Sedgwick, S. D. Bull, X.-P. He, H. Tian, J. Yoon, J. L. Sessler and T. D. James, Chem. Soc. Rev., 2020, 49, 5110-5139.

107 L. Li, R. Guan, M. Guo, P. Ning, R. Shao and X. Meng, Sens. Actuators, B, 2018, 254, 949-955.

108 X. Zhang, Y. Xiao and X. Qian, Angew. Chem., Int. Ed., 2008, 47, 8025-8029.

109 K. Aich, S. Goswami, S. Das, C. D. Mukhopadhyay, C. K. Quah and H.-K. Fun, Inorg. Chem., 2015, 54, 7309-7315.

110 H. Xu, C. Zhu, Y. Chen, Y. Bai, Z. Han, S. Yao, Y. Jiao, H. Yuan, W. He and Z. Guo, Chem. Sci., 2020, 11, 11037-11041.

111 Y. Ma, H. Chen, F. Wang, S. Kambam, Y. Wang, C. Mao and X. Chen, Dyes Pigm., 2014, 102, 301-307.

112 D. Cao, L. Zhu, Z. Liu and W. Lin, J. Photochem. Photobiol., C, 2020, 44, 100371.

113 L. Zhou, X. Zhang, Q. Wang, Y. Lv, G. Mao, A. Luo, Y. Wu, Y. Wu, J. Zhang and W. Tan, J. Am. Chem. Soc., 2014, 136, 9838-9841.

114 Y. Nakahara, T. Kida, Y. Nakatsuji and M. Akashi, Chem. Commun., 2004, 224-225.

115 F. Li, K. Zhong, S. Hou, L. Tang and Y. Bian, Tetrahedron Lett., 2020, 61, 151558.

116 K. Chaichana, N. Phutlaprungrueang, L. Chaicharoenwimolkul, M. Promkatkaew and S. Kongsriprapan, Spectrochim. Acta, Part A, 2019, 207, 118-122.

117 P. Saluja, N. Kaur, N. Singh and D. O. Jang, Tetrahedron Lett., 2011, 52, 6705-6708.

118 A. K. K. Bhasin, P. Chauhan and S. Chaudhary, Sens. Actuators, B, 2019, 294, 116-122.

119 P. N. Basa, A. Bhowmick, M. M. Schulz and A. G. Sykes, J. Org. Chem., 2011, 76, 7866-7871.

120 Y. Nakahara, T. Kida, Y. Nakatsuji and M. Akashi, Org. Biomol. Chem., 2005, 3, 1787-1794.

121 I. Rivilla, B. Aparicio, J. M. Bueno, D. Casanova, C. Tonnelé, Z. Freixa, P. Herrero, C. Rogero, J. I. Miranda, R. M. Martínez-Ojeda, F. Monrabal, B. Olave, T. Schäfer, P. Artal, D. Nygren, F. P. Cossío and J. J. Gómez-Cadenas, Nature, 2020, 583, 48-54. 